TTK-10-42

SI-HEP-2010-10

TTP10-32

$\mathrm{SFB} / \mathrm{CPP}-10-63$

October 23, 2018

\title{
Heavy-to-light currents at NNLO in SCET and semi-inclusive $\bar{B} \rightarrow X_{s} \ell^{+} \ell^{-}$decay
}

\author{
G. Bell ${ }^{a}$, M. Beneke ${ }^{b}$, T. Huber ${ }^{c}$ and Xin-Qiang Li ${ }^{d}, e$ \\ a Albert Einstein Center for Fundamental Physics, \\ Institute for Theoretical Physics, University of Bern, \\ Sidlerstrasse 5, 3012 Bern, Switzerland \\ ${ }^{b}$ Institut für Theoretische Teilchenphysik und Kosmologie, \\ RWTH Aachen University, \\ D-52056 Aachen, Germany \\ ${ }^{c}$ Theoretische Physik 1, Fachbereich 7, \\ Universität Siegen, D-57068 Siegen, Germany \\ ${ }^{d}$ Department of Physics, Henan Normal University, \\ Xinxiang, Henan 453007, P. R. China \\ e Institute of Theoretical Physics, \\ Chinese Academy of Science, \\ Beijing 100190, P. R. China
}

\begin{abstract}
We perform the two-loop matching calculation for heavy-to-light currents from QCD onto soft-collinear effective theory for the complete set of Dirac structures. The newly obtained matching coefficients enter several phenomenological applications, of which we discuss heavy-to-light form factor ratios and exclusive radiative decays, as well as the semi-inclusive decay $\bar{B} \rightarrow X_{s} \ell^{+} \ell^{-}$. For this decay, we observe a significant shift of the forward-backward asymmetry zero and find $q_{0}^{2}=\left(3.34_{-0.25}^{+0.22}\right) \mathrm{GeV}^{2}$ for an invariant mass cut $m_{X}^{\text {cut }}=2.0 \mathrm{GeV}$.
\end{abstract}




\section{Introduction}

The flavour-changing quark currents $\bar{q} \Gamma_{i} b$, with $\Gamma_{i}=\left\{1, \gamma_{5}, \gamma^{\mu}, \gamma_{5} \gamma^{\mu}, i \sigma^{\mu \nu}\right\}$, govern the hadronic dynamics in semi-leptonic and radiative $B$ decays. The matrix elements of the currents, usually parameterized by several transition form factors, are also important inputs to the factorization formulae for non-leptonic $B$ decays [1. In the kinematic region where the hadronic final state has small invariant mass but large energy, softcollinear effective theory (SCET) [2,3] is the appropriate theoretical framework, with which transparent factorization formulae for the heavy-to-light form factors have been derived [4] (see also [5,6]). Thus, the accurate representation of the heavy-to-light currents in SCET is of particular interest.

The LO and NLO matching coefficients for heavy-to-light currents from QCD onto SCET for an arbitrary Dirac matrix has been worked out a few years ago [2, 7, 8, The coefficients for V-A currents have recently been determined to NNLO in the context of inclusive semi-leptonic $B$ decays [9-12] in the shape-function region. In this paper we complete the NNLO calculation by computing the remaining matching coefficients of the tensor currents. The tensor matching coefficients enter several phenomenological applications, of which we shall discuss heavy-to-light form factor ratios and exclusive radiative decays, as well as the semi-inclusive decay $\bar{B} \rightarrow X_{s} \ell^{+} \ell^{-}$.

The paper is organized as follows. In Section 2 we first set up notation and then briefly recapitulate the techniques applied and the necessary ingredients for the twoloop calculation. In Section 3 the two-loop calculation of the QCD form factors and the corresponding matching coefficients are presented in detail. Three interesting phenomenological applications of our results to heavy-to-light form factor ratios, exclusive radiative decays, as well as the inclusive decay $\bar{B} \rightarrow X_{s} \ell^{+} \ell^{-}$are discussed in Sections 4 and 5. We conclude in Section 6. The lengthy analytic expressions for the coefficient functions can be found in Appendices A and B.

\section{NNLO calculation}

\subsection{Set-up of the matching calculation}

A generic heavy-to-light current $\bar{q} \Gamma_{i} b$ is represented in SCET by a set of non-local "twobody" and "three-body" [3-6] operators,

$$
\begin{aligned}
{\left[\bar{q} \Gamma_{i} b\right](0)=} & \sum_{j} \int d s \tilde{C}_{i}^{j}(s)\left[\bar{\xi} W_{h c}\right]\left(s n_{+}\right) \Gamma_{j}^{\prime} h_{v}(0) \\
& +\sum_{j} \int d s_{1} d s_{2} \tilde{C}_{i \mu}^{(B 1) j}\left(s_{1}, s_{2}\right) O_{i}^{(B 1) j \mu}\left(s_{1}, s_{2}\right)+\ldots,
\end{aligned}
$$

where $h_{v}$ is the static heavy quark field defined in HQET, whereas $\xi$ and $W_{h c}$ are the hard-collinear light quark field and a hard-collinear Wilson line from SCET, respectively. In this paper we are concerned with the calculation of the matching coefficients in the 
first line of (11). The three-body operators $O_{i}^{(B 1) j \mu}\left(s_{1}, s_{2}\right)$ in the second line are $1 / m_{b^{-}}$ suppressed but relevant at leading power for exclusive transitions and form factors due to the matrix element suppression of the leading term. Their one-loop matching coefficients are known from [8, 13] and this suffices to work out their contribution to the exclusive transitions at $\mathcal{O}\left(\alpha_{s}^{2}\right)$. We refer to [14] for the details of the calculation of these spectator-scattering terms. In the current work we consider the missing $\mathcal{O}\left(\alpha_{s}^{2}\right)$ matching coefficients of the two-body operators $\left[\bar{\xi} W_{h c}\right]\left(s n_{+}\right) \Gamma_{j}^{\prime} h_{v}(0)$ and adopt the momentum space representation, which follows from

$$
C_{i}^{j}\left(n_{+} p\right)=\int d s e^{i s n_{+} p} \tilde{C}_{i}^{j}(s)
$$

We decompose the heavy-to-light currents in the basis from [14] (summarized in Table 1) with two reference vectors $v$ and $n_{-}$that fulfill $v=\left(n_{-}+n_{+}\right) / 2, n_{ \pm}^{2}=0$ and $n_{+} n_{-}=2$. The matching calculation involves 12 coefficient functions $C_{i}^{j}$, which are not independent in a renormalization scheme with anti-commuting $\gamma_{5}$ due to the chiral symmetry of QCD. In the NDR scheme adopted in this work, this translates into the constraints $C_{P}=C_{S}$ and $C_{A}^{i}=C_{V}^{i}$, while similar relations hold between the matching coefficients of the tensor and the pseudotensor current. As the latter is reducible in four space-time dimensions, we obtain the additional constraints $C_{T}^{2}=C_{T}^{4}=0$ in four dimensions. We nevertheless keep the more general basis from Table 1, since we work in dimensional regularization and obtain intermediate results that are valid in $d=4-2 \epsilon \operatorname{dimensions,~where~} C_{T}^{2}$ and $C_{T}^{4}$ are of $\mathcal{O}(\epsilon)$ but non-vanishing.

It is convenient to perform the matching calculation with on-shell quarks and to use dimensional regularization to regularize ultraviolet (UV) and infrared (IR) singularities. The SCET diagrams are then scaleless and vanish and the computation essentially amounts to a two-loop calculation in QCD. We, in particular, introduce an analogous tensor decomposition to (11) and parameterize the QCD result in terms of 12 form factors,

$$
\left\langle q(p)\left|\bar{q} \Gamma_{i} b\right| b\left(p_{b}\right)\right\rangle=\sum_{j} F_{i}^{j}\left(q^{2}\right) \bar{u}(p) \Gamma_{j}^{\prime} u\left(p_{b}\right)
$$

where $p_{b}=m_{b} v$ is the momentum of the heavy quark, $p=u m_{b} n_{-} / 2$ the momentum of the light quark and $q^{2}=\left(p_{b}-p\right)^{2}=(1-u) m_{b}^{2}$ denotes the momentum transfer. Due to the absence of loop contributions on the effective theory side, the SCET matrix elements are given by the tree level matrix elements multiplied by a universal renormalization factor $Z_{J}$ of the SCET current $\left[\bar{\xi} W_{h c}\right] \Gamma_{j}^{\prime} h_{v}$. There is thus a one-to-one correspondence

\begin{tabular}{|c|c|c|c|c|c|c|c|c|c|c|c|c|}
\hline$\Gamma_{i}$ & 1 & $\gamma_{5}$ & \multicolumn{3}{|c|}{$\gamma^{\mu}$} & \multicolumn{4}{|c|}{$\gamma_{5} \gamma^{\mu}$} & \multicolumn{4}{|c|}{$i \sigma^{\mu \nu}$} \\
\hline$\Gamma_{j}^{\prime}$ & 1 & $\gamma_{5}$ & $\gamma^{\mu}$ & $v^{\mu}$ & $n_{-}^{\mu}$ & $\gamma_{5} \gamma^{\mu}$ & $v^{\mu} \gamma_{5}$ & $n_{-}^{\mu} \gamma_{5}$ & $\gamma^{[\mu} \gamma^{\nu]}$ & $v^{[\mu} \gamma^{\nu]}$ & $n_{-}^{[\mu} \gamma^{\nu]}$ & $n_{-}^{[\mu} v^{\nu]}$ \\
\hline$C_{i}^{j}$ & $C_{S}$ & $C_{P}$ & $C_{V}^{1}$ & $C_{V}^{2}$ & $C_{V}^{3}$ & $C_{A}^{1}$ & $C_{A}^{2}$ & $C_{A}^{3}$ & $C_{T}^{1}$ & $C_{T}^{2}$ & $C_{T}^{3}$ & $C_{T}^{4}$ \\
\hline
\end{tabular}

Table 1: Matching coefficients $C_{i}^{j}$ according to the decomposition in (1) (a $\left.a^{[\mu} b^{\nu]} \equiv a^{\mu} b^{\nu}-a^{\nu} b^{\mu}\right)$. 
between the matching coefficients $C_{i}^{j}$ and the form factors $F_{i}^{j}$,

$$
C_{i}^{j}=Z_{J}^{-1} F_{i}^{j} .
$$

As the form factors are, however, in general IR-divergent, there exists no analogous relation on the form factor level to the four-dimensional constraints $C_{T}^{2}=C_{T}^{4}=0$.

The purpose of our analysis consists in the computation of the matching coefficients

$C_{i}^{j}$ (and the respective form factors $F_{i}^{j}$ ) to NNLO in QCD. Whereas the NLO corrections have been worked out in $\left[2,7,8,8\right.$, the coefficients $C_{V}^{i}$ and $C_{A}^{i}$ have recently been determined to NNLO in the context of inclusive semi-leptonic $B$ decays [12]. In the current work we complete the NNLO calculation by computing the remaining matching coefficients $C_{S}$, $C_{P}$ and $C_{T}^{i}$. The four-dimensional constraints mentioned above, will serve as a non-trivial check of our calculation.

\subsection{Technical aspects of the calculation}

We organize the calculation along the strategy that we used in our previous works on the V-A current [11,12]. The calculation is based on an automated reduction algorithm, which uses integration-by-parts techniques [15] and the Laporta algorithm [16] to express the two-loop diagrams (shown in Figure 1 of [11]) in terms of a small set of scalar master integrals. The required master integrals are already known from the computations in 9 11, 17, 18.

Our results will be given in terms of the following set of harmonic polylogarithms (HPLs) [19],

$$
\begin{aligned}
H(0 ; x) & =\ln (x), & H(0,0,1 ; x) & =\mathrm{Li}_{3}(x), \\
H(1 ; x) & =-\ln (1-x), & H(0,1,1 ; x) & =\mathrm{S}_{1,2}(x), \\
H(-1 ; x) & =\ln (1+x), & H(0,0,0,1 ; x) & =\mathrm{Li}_{4}(x), \\
H(0,1 ; x) & =\mathrm{Li}_{2}(x), & H(0,0,1,1 ; x) & =\mathrm{S}_{2,2}(x), \\
H(0,-1 ; x) & =-\mathrm{Li}_{2}(-x), & H(0,1,1,1 ; x) & =\mathrm{S}_{1,3}(x), \\
H(-1,0,1 ; x) & \equiv \mathcal{H}_{1}(x), & H(0,-1,0,1 ; x) & \equiv \mathcal{H}_{2}(x),
\end{aligned}
$$

where we introduced a shorthand notation for the last two HPLs. Whereas the first one can be written in a compact form [20],

$$
\mathcal{H}_{1}(x)=\ln (1+x) \operatorname{Li}_{2}(x)+\frac{1}{2} \mathrm{~S}_{1,2}\left(x^{2}\right)-\mathrm{S}_{1,2}(x)-\mathrm{S}_{1,2}(-x),
$$

the second one, $\mathcal{H}_{2}(x)=\int_{0}^{x} d x^{\prime} \mathcal{H}_{1}\left(x^{\prime}\right) / x^{\prime}$, cannot be expressed in terms of Nielsen Polylogarithms and has to be evaluated numerically.

The charm quark enters the matching calculation at the two-loop level through the gluon self energy which contains closed fermion loops. Our analytical results from Sections 3.1 and 3.2 are valid for massless charm quark, but we also show some numerical 
results in Section 3.2 that include charm mass effects. In this case we formally keep $m_{c} / m_{b}$ fixed in the heavy-quark expansion, so the coefficients depend non-trivially on the quark mass ratio (see Section 5 of [12]).

The pure two-loop calculation yields bare form factors $F_{i}^{j}$ that are UV- and IRdivergent. The UV-divergences are subtracted in a standard renormalization procedure, which has been described in detail in our previous works [11,12]. We, in particular, renormalize the strong coupling constant in the $\overline{\mathrm{MS}}$-scheme, whereas the quark wave-functions and the $b$-quark mass are renormalized in the on-shell scheme. The only difference in the current calculation consists in the fact that the scalar and the tensor current have nonvanishing anomalous dimensions in contrast to the vector current considered in [11,12]. This gives rise to an additional multiplicative counterterm $Z_{i}^{-1}(i=S, T)$. We expand the inverse

$$
Z_{i}=1+\sum_{k=1}^{\infty}\left(\frac{\alpha_{s}^{(5)}}{4 \pi}\right)^{k} Z_{i}^{(k)}
$$

in terms of the renormalized coupling constant of a theory with five active quark flavours. In the $\overline{\mathrm{MS}}$-scheme the respective NLO coefficients are then given by $Z_{S}^{(1)}=3 C_{F} / \epsilon$ and $Z_{T}^{(1)}=-C_{F} / \epsilon$ for the scalar and the tensor current, respectively. At NNLO the counterterms can be inferred from [21],

$$
\begin{aligned}
& Z_{S}^{(2)}=C_{F}\left\{\left[\frac{9}{2} C_{F}-\frac{11}{2} C_{A}+2 n_{f} T_{F}\right] \frac{1}{\epsilon^{2}}+\left[\frac{3}{4} C_{F}+\frac{97}{12} C_{A}-\frac{5}{3} n_{f} T_{F}\right] \frac{1}{\epsilon}\right\}, \\
& Z_{T}^{(2)}=C_{F}\left\{\left[\frac{1}{2} C_{F}+\frac{11}{6} C_{A}-\frac{2}{3} n_{f} T_{F}\right] \frac{1}{\epsilon^{2}}+\left[\frac{19}{4} C_{F}-\frac{257}{36} C_{A}+\frac{13}{9} n_{f} T_{F}\right] \frac{1}{\epsilon}\right\},
\end{aligned}
$$

where $n_{f}=5$ denotes the number of active quark flavours.

\section{Results}

\subsection{Renormalized form factors}

We first present our results for the renormalized form factors $F_{i}^{j}$, which are UV-finite but IR-divergent. It will be convenient to decompose the form factors according to

$$
F_{i}^{j}=\sum_{k=0}^{\infty}\left(\frac{\alpha_{s}^{(5)}}{4 \pi}\right)^{k} F_{i}^{j,(k)}, \quad F_{i}^{j,(k)}=\sum_{l} F_{i, l}^{j,(k)} \epsilon^{l} .
$$

In this normalization the form factors become at tree level

$$
\begin{aligned}
& F_{S}^{(0)}=-2 F_{T}^{1,(0)}=1, \\
& F_{T}^{2,(0)}=F_{T}^{3,(0)}=F_{T}^{4,(0)}=0 .
\end{aligned}
$$


Here and below we do not quote our results for the pseudoscalar and the (axial) vector current, since the former are equal to those of the scalar current in the NDR scheme, while the latter have already been computed before and can be found in [9-12].

One-loop form factors. At NLO we compute the form factors up to terms of $\mathcal{O}\left(\epsilon^{2}\right)$. Our results are given in terms of a set of coefficient functions $g_{i}(u)$, which we list in Appendix A. The scalar form factor is IR-divergent and becomes (with $q^{2}=\bar{u} m_{b}^{2}$, $\bar{u}=1-u$ and $L=\ln \mu^{2} / m_{b}^{2}$ ),

$$
\begin{aligned}
F_{S,-2}^{(1)}(u) & =-C_{F}, \\
F_{S,-1}^{(1)}(u) & =C_{F}\left(g_{0}(u)-L\right), \\
F_{S, 0}^{(1)}(u) & =C_{F}\left(g_{1}(u)+\left[g_{0}(u)+3\right] L-\frac{1}{2} L^{2}\right), \\
F_{S, 1}^{(1)}(u) & =C_{F}\left(g_{2}(u)+g_{1}(u) L+\frac{1}{2}\left[g_{0}(u)+3\right] L^{2}-\frac{1}{6} L^{3}\right), \\
F_{S, 2}^{(1)}(u) & =C_{F}\left(g_{3}(u)+g_{2}(u) L+\frac{1}{2} g_{1}(u) L^{2}+\frac{1}{6}\left[g_{0}(u)+3\right] L^{3}-\frac{1}{24} L^{4}\right) .
\end{aligned}
$$

The first tensor form factor is also IR-divergent and given by

$$
\begin{aligned}
& F_{T,-2}^{1,(1)}(u)=\frac{C_{F}}{2}, \\
& F_{T,-1}^{1,(1)}(u)=-\frac{C_{F}}{2}\left(g_{0}(u)-L\right), \\
& F_{T, 0}^{1,(1)}(u)=-\frac{C_{F}}{2}\left(g_{4}(u)+\left[g_{0}(u)-1\right] L-\frac{1}{2} L^{2}\right), \\
& F_{T, 1}^{1,(1)}(u)=-\frac{C_{F}}{2}\left(g_{5}(u)+g_{4}(u) L+\frac{1}{2}\left[g_{0}(u)-1\right] L^{2}-\frac{1}{6} L^{3}\right), \\
& F_{T, 2}^{1,(1)}(u)=-\frac{C_{F}}{2}\left(g_{6}(u)+g_{5}(u) L+\frac{1}{2} g_{4}(u) L^{2}+\frac{1}{6}\left[g_{0}(u)-1\right] L^{3}-\frac{1}{24} L^{4}\right),
\end{aligned}
$$

whereas the other tensor form factors are IR-finite at NLO and read

$$
\begin{aligned}
& F_{T, 0}^{2,(1)}(u)=0 \\
& F_{T, 1}^{2,(1)}(u)=C_{F} g_{7}(u), \\
& F_{T, 2}^{2,(1)}(u)=C_{F}\left(g_{8}(u)+g_{7}(u) L\right), \\
& F_{T, 0}^{3,(1)}(u)=C_{F} g_{9}(u),
\end{aligned}
$$




$$
\begin{aligned}
& F_{T, 1}^{3,(1)}(u)=C_{F}\left(g_{10}(u)+g_{9}(u) L\right) \\
& F_{T, 2}^{3,(1)}(u)=C_{F}\left(g_{11}(u)+g_{10}(u) L+\frac{1}{2} g_{9}(u) L^{2}\right) \\
& F_{T, 0}^{4,(1)}(u)=0 \\
& F_{T, 1}^{4,(1)}(u)=C_{F} g_{12}(u) \\
& F_{T, 2}^{4,(1)}(u)=C_{F}\left(g_{13}(u)+g_{12}(u) L\right)
\end{aligned}
$$

Two-loop form factors. At NNLO the IR-divergent parts of the form factors can be expressed in terms of the one-loop coefficient functions $g_{i}(u)$. The divergent terms of the scalar form factor read

$$
\begin{aligned}
F_{S,-4}^{(2)}(u)= & \frac{1}{2} C_{F}^{2} \\
F_{S,-3}^{(2)}(u)= & C_{F}^{2}\left(L-g_{0}(u)\right)+\frac{11}{4} C_{A} C_{F}-n_{l} T_{F} C_{F} \\
F_{S,-2}^{(2)}(u)= & C_{F}^{2}\left[L^{2}-\left(2 g_{0}(u)+3\right) L+\frac{1}{2} g_{0}(u)^{2}-g_{1}(u)\right]+\frac{4}{3} L T_{F} C_{F} \\
& +C_{A} C_{F}\left[\frac{11}{6}\left(L-g_{0}(u)\right)-\frac{67}{36}+\frac{\pi^{2}}{12}\right]+n_{l} T_{F} C_{F}\left[\frac{5}{9}-\frac{2}{3}\left(L-g_{0}(u)\right)\right] \\
F_{S,-1}^{(2)}(u)= & C_{F}^{2}\left[\frac{2}{3} L^{3}-\left(2 g_{0}(u)+\frac{9}{2}\right) L^{2}-\left(2 g_{1}(u)-g_{0}(u)^{2}-3 g_{0}(u)\right) L\right. \\
& \left.+g_{0}(u) g_{1}(u)-g_{2}(u)-\frac{3}{8}+\frac{\pi^{2}}{2}-6 \zeta_{3}\right] \\
& +C_{A} C_{F}\left[\left(\frac{\pi^{2}}{6}-\frac{67}{18}\right)\left(L-g_{0}(u)\right)+\frac{461}{216}-\frac{17 \pi^{2}}{24}+\frac{11}{2} \zeta_{3}\right] \\
& +n_{l} T_{F} C_{F}\left[\frac{10}{9}\left(L-g_{0}(u)\right)-\frac{25}{54}+\frac{\pi^{2}}{6}\right]+T_{F} C_{F}\left[2 L^{2}-\frac{4}{3} g_{0}(u) L+\frac{\pi^{2}}{9}\right]
\end{aligned}
$$

and for the first tensor form factor we get

$$
\begin{aligned}
& F_{T,-4}^{1,(2)}(u)=-\frac{1}{4} C_{F}^{2} \\
& F_{T,-3}^{1,(2)}(u)=-\frac{1}{2} C_{F}^{2}\left(L-g_{0}(u)\right)-\frac{11}{8} C_{A} C_{F}+\frac{1}{2} n_{l} T_{F} C_{F} \\
& F_{T,-2}^{1,(2)}(u)=-\frac{1}{2} C_{F}^{2}\left[L^{2}-\left(2 g_{0}(u)-1\right) L+\frac{1}{2} g_{0}(u)^{2}-g_{4}(u)\right]-\frac{2}{3} L T_{F} C_{F}
\end{aligned}
$$




$$
\begin{aligned}
& -\frac{1}{2} C_{A} C_{F}\left[\frac{11}{6}\left(L-g_{0}(u)\right)-\frac{67}{36}+\frac{\pi^{2}}{12}\right]-\frac{1}{2} n_{l} T_{F} C_{F}\left[\frac{5}{9}-\frac{2}{3}\left(L-g_{0}(u)\right)\right], \\
F_{T,-1}^{1,(2)}(u)= & -\frac{1}{2} C_{F}^{2}\left[\frac{2}{3} L^{3}-\left(2 g_{0}(u)-\frac{3}{2}\right) L^{2}-\left(2 g_{4}(u)-g_{0}(u)^{2}+g_{0}(u)\right) L\right. \\
& \left.+g_{0}(u) g_{4}(u)-g_{5}(u)-\frac{3}{8}+\frac{\pi^{2}}{2}-6 \zeta_{3}\right] \\
& -\frac{1}{2} C_{A} C_{F}\left[\left(\frac{\pi^{2}}{6}-\frac{67}{18}\right)\left(L-g_{0}(u)\right)+\frac{461}{216}-\frac{17 \pi^{2}}{24}+\frac{11}{2} \zeta_{3}\right] \\
& -\frac{1}{2} n_{l} T_{F} C_{F}\left[\frac{10}{9}\left(L-g_{0}(u)\right)-\frac{25}{54}+\frac{\pi^{2}}{6}\right]-\frac{1}{2} T_{F} C_{F}\left[2 L^{2}-\frac{4}{3} g_{0}(u) L+\frac{\pi^{2}}{9}\right] .
\end{aligned}
$$

The IR-divergent parts of the other tensor form factors are given by

$$
F_{T,-1}^{2,(2)}(u)=-C_{F}^{2} g_{7}(u)
$$

and

$$
\begin{aligned}
& F_{T,-2}^{3,(2)}(u)=-C_{F}^{2} g_{9}(u) \\
& F_{T,-1}^{3,(2)}(u)=C_{F}^{2}\left(g_{0}(u) g_{9}(u)-g_{10}(u)-2 g_{9}(u) L\right)
\end{aligned}
$$

and

$$
F_{T,-1}^{4,(2)}(u)=-C_{F}^{2} g_{12}(u)
$$

The finite parts of the two-loop form factors involve a new set of coefficient functions $h_{i}(u)$, which we specify in Appendix B. We find

$$
\begin{aligned}
F_{S, 0}^{(2)}(u)=C_{F}^{2}[ & \frac{1}{3} L^{4}-\left(\frac{4}{3} g_{0}(u)+\frac{7}{2}\right) L^{3}-\left(2 g_{1}(u)-g_{0}(u)^{2}-\frac{9}{2} g_{0}(u)-\frac{9}{2}\right) L^{2} \\
& \left.-\left(2 g_{2}(u)-2 g_{1}(u) g_{0}(u)-3 g_{1}(u)-\frac{3}{4}-\pi^{2}+12 \zeta_{3}\right) L+h_{1}(u)\right] \\
+ & C_{A} C_{F}\left[-\frac{11}{18} L^{3}+\left(\frac{11}{6} g_{0}(u)+\frac{16}{9}+\frac{\pi^{2}}{6}\right) L^{2}\right. \\
& \left.+\left(\frac{11}{3} g_{1}(u)+\left(\frac{67}{9}-\frac{\pi^{2}}{3}\right) g_{0}(u)+\frac{2207}{108}-\frac{17 \pi^{2}}{12}+11 \zeta_{3}\right) L+h_{2}(u)\right] \\
+ & n_{l} T_{F} C_{F}\left[\frac{2}{9} L^{3}-\left(\frac{2}{3} g_{0}(u)+\frac{8}{9}\right) L^{2}-\left(\frac{4}{3} g_{1}(u)+\frac{20}{9} g_{0}(u)+\frac{115}{27}-\frac{\pi^{2}}{3}\right) L\right. \\
& \left.-\frac{4}{3} g_{2}(u)-\frac{20}{9} g_{1}(u)-\left(\frac{20}{27}+\frac{\pi^{2}}{3}\right) g_{0}(u)-\frac{541}{324}-\frac{13 \pi^{2}}{18}+\frac{10}{3} \zeta_{3}\right]
\end{aligned}
$$




$$
+T_{F} C_{F}\left[\frac{14}{9} L^{3}-\left(2 g_{0}(u)+2\right) L^{2}-\left(\frac{4}{3} g_{1}(u)+\frac{10}{3}-\frac{2 \pi^{2}}{9}\right) L+h_{3}(u)\right]
$$

and

$$
\begin{aligned}
F_{T, 0}^{1,(2)}(u)=-\frac{1}{2} C_{F}^{2}\left[\frac{1}{3} L^{4}-\left(\frac{4}{3} g_{0}(u)-\frac{7}{6}\right) L^{3}-\left(2 g_{4}(u)-g_{0}(u)^{2}+\frac{3}{2} g_{0}(u)-\frac{1}{2}\right) L^{2}\right. \\
\left.-\left(2 g_{5}(u)-2 g_{4}(u) g_{0}(u)+g_{4}(u)-\frac{35}{4}-\pi^{2}+12 \zeta_{3}\right) L+h_{4}(u)\right] \\
-\frac{1}{2} C_{A} C_{F}\left[-\frac{11}{18} L^{3}+\left(\frac{11}{6} g_{0}(u)-\frac{50}{9}+\frac{\pi^{2}}{6}\right) L^{2}\right. \\
\left.\quad+\left(\frac{11}{3} g_{4}(u)+\left(\frac{67}{9}-\frac{\pi^{2}}{3}\right) g_{0}(u)-\frac{1081}{108}-\frac{17 \pi^{2}}{12}+11 \zeta_{3}\right) L+h_{5}(u)\right] \\
-\frac{1}{2} n_{l} T_{F} C_{F}\left[\frac{2}{9} L^{3}-\left(\frac{2}{3} g_{0}(u)-\frac{16}{9}\right) L^{2}-\left(\frac{4}{3} g_{4}(u)+\frac{20}{9} g_{0}(u)-\frac{53}{27}-\frac{\pi^{2}}{3}\right) L\right. \\
\left.\quad-\frac{4}{3} g_{5}(u)-\frac{20}{9} g_{4}(u)-\left(\frac{20}{27}+\frac{\pi^{2}}{3}\right) g_{0}(u)+h_{6}(u)\right] \\
-\frac{1}{2} T_{F} C_{F}\left[\frac{14}{9} L^{3}-\left(2 g_{0}(u)-\frac{2}{3}\right) L^{2}-\left(\frac{4}{3} g_{4}(u)-\frac{26}{9}-\frac{2 \pi^{2}}{9}\right) L+h_{7}(u)\right],
\end{aligned}
$$

and

$$
F_{T, 0}^{2,(2)}(u)=C_{F}^{2}\left(g_{0}(u) g_{7}(u)-g_{8}(u)-2 g_{7}(u) L\right)
$$

and

$$
\begin{aligned}
F_{T, 0}^{3,(2)}(u)= & C_{F}^{2}\left[-2 g_{9}(u) L^{2}+\left(2 g_{0}(u) g_{9}(u)-g_{9}(u)-2 g_{10}(u)\right) L+h_{8}(u)\right] \\
& +C_{A} C_{F}\left[\frac{11}{3} g_{9}(u) L+h_{9}(u)\right]+T_{F} C_{F}\left[-\frac{4}{3} g_{9}(u) L+h_{10}(u)\right] \\
& +n_{l} T_{F} C_{F}\left[-\frac{4}{3} g_{9}(u) L-\frac{4}{3} g_{10}(u)-\frac{8}{9} g_{9}(u)+\frac{4 u}{3 \bar{u}^{2}} \ln (u)+\frac{4 u}{3 \bar{u}}\right]
\end{aligned}
$$

and

$$
F_{T, 0}^{4,(2)}(u)=C_{F}^{2}\left(g_{0}(u) g_{12}(u)-g_{13}(u)-2 g_{12}(u) L\right)
$$

\subsection{Matching coefficients}

The matching coefficients $C_{i}^{j}$ follow from the above expressions for the renormalized form

factors $F_{i}^{j}$ after multiplication with the inverse of the renormalization factor of the SCET 
current $Z_{J}$, cf. (4). To this end one has to keep in mind that the form factors have been computed in QCD with five active quark flavours, while $Z_{J}$ is usually given in SCET with four active flavours. We thus have

$$
Z_{J}=1+\sum_{k=1}^{\infty}\left(\frac{\alpha_{s}^{(4)}}{4 \pi}\right)^{k} Z_{J}^{(k)}
$$

with NLO coefficient [2],

$$
Z_{J}^{(1)}=C_{F}\left\{-\frac{1}{\epsilon^{2}}-\frac{1}{\epsilon}\left(\ln \frac{\mu^{2}}{u^{2} m_{b}^{2}}+\frac{5}{2}\right)\right\} .
$$

The two-loop anomalous dimension can be deduced from [22] (see also [10])

$$
\begin{aligned}
Z_{J}^{(2)}= & C_{F}\left\{\frac{C_{F}}{2 \epsilon^{4}}+\left[\left(\ln \frac{\mu^{2}}{u^{2} m_{b}^{2}}+\frac{5}{2}\right) C_{F}+\frac{11}{4} C_{A}-n_{l} T_{F}\right] \frac{1}{\epsilon^{3}}\right. \\
& +\left[\frac{1}{2}\left(\ln \frac{\mu^{2}}{u^{2} m_{b}^{2}}+\frac{5}{2}\right)^{2} C_{F}+\left(\frac{\pi^{2}}{12}-\frac{67}{36}+\frac{11}{6}\left(\ln \frac{\mu^{2}}{u^{2} m_{b}^{2}}+\frac{5}{2}\right)\right) C_{A}\right. \\
& \left.+\left(\frac{5}{9}-\frac{2}{3}\left(\ln \frac{\mu^{2}}{u^{2} m_{b}^{2}}+\frac{5}{2}\right)\right) n_{l} T_{F}\right] \frac{1}{\epsilon^{2}}+\left[\left(\frac{\pi^{2}}{2}-\frac{3}{8}-6 \zeta_{3}\right) C_{F}\right. \\
& +\left(\frac{461}{216}-\frac{17 \pi^{2}}{24}+\frac{11}{2} \zeta_{3}+\left(\frac{\pi^{2}}{6}-\frac{67}{18}\right)\left(\ln \frac{\mu^{2}}{u^{2} m_{b}^{2}}+\frac{5}{2}\right)\right) C_{A} \\
& \left.\left.+\left(\frac{\pi^{2}}{6}-\frac{25}{54}+\frac{10}{9}\left(\ln \frac{\mu^{2}}{u^{2} m_{b}^{2}}+\frac{5}{2}\right)\right) n_{l} T_{F}\right] \frac{1}{\epsilon}\right\},
\end{aligned}
$$

where $n_{l}=n_{f}-1=4$ is the number of active quark flavours in the effective theory.

We now expand the matching coefficients in terms of the coupling constant of the four-flavour theory as

$$
C_{i}^{j}=\sum_{k=0}^{\infty}\left(\frac{\alpha_{s}^{(4)}}{4 \pi}\right)^{k} C_{i}^{j,(k)}
$$

and rewrite (4) up to NNLO, which yields

$$
\begin{aligned}
& C_{i}^{j,(0)}=F_{i}^{j,(0)} \\
& C_{i}^{j,(1)}=F_{i}^{j,(1)}-Z_{J}^{(1)} F_{i}^{j,(0)} \\
& C_{i}^{j,(2)}=F_{i}^{j,(2)}+\delta \alpha_{s}^{(1)} F_{i}^{j,(1)}-Z_{J}^{(1)}\left(F_{i}^{j,(1)}-Z_{J}^{(1)} F_{i}^{j,(0)}\right)-Z_{J}^{(2)} F_{i}^{j,(0)} .
\end{aligned}
$$

Notice that the last relation implies a term which stems from the conversion of the fiveflavour to the four-flavour coupling constant,

$$
\alpha_{s}^{(5)}=\alpha_{s}^{(4)}\left[1+\frac{\alpha_{s}^{(4)}}{4 \pi} \delta \alpha_{s}^{(1)}+\mathcal{O}\left(\alpha_{s}^{2}\right)\right]
$$


with (see also [11,12] for further details)

$$
\delta \alpha_{s}^{(1)}=T_{F}\left[\frac{4}{3} \ln \frac{\mu^{2}}{m_{b}^{2}}+\left(\frac{2}{3} \ln ^{2} \frac{\mu^{2}}{m_{b}^{2}}+\frac{\pi^{2}}{9}\right) \epsilon+\left(\frac{2}{9} \ln ^{3} \frac{\mu^{2}}{m_{b}^{2}}+\frac{\pi^{2}}{9} \ln \frac{\mu^{2}}{m_{b}^{2}}-\frac{4}{9} \zeta_{3}\right) \epsilon^{2}+\mathcal{O}\left(\epsilon^{3}\right)\right] .
$$

At LO the matching coefficients then become

$$
\begin{aligned}
& C_{S}^{(0)}=-2 C_{T}^{1,(0)}=1, \\
& C_{T}^{2,(0)}=C_{T}^{3,(0)}=C_{T}^{4,(0)}=0 .
\end{aligned}
$$

At NLO the matching coefficients are given by the finite terms of the one-loop form factors,

$$
\begin{aligned}
C_{S}^{(1)}(u) & =F_{S, 0}^{(1)}(u), \\
C_{T}^{1,(1)}(u) & =F_{T, 0}^{1,(1)}(u), \\
C_{T}^{3,(1)}(u) & =F_{T, 0}^{3,(1)}(u),
\end{aligned}
$$

and, in particular, $C_{T}^{2,(1)}=F_{T, 0}^{2,(1)}=0$ and $C_{T}^{4,(1)}=F_{T, 0}^{4,(1)}=0$ in accordance with the four-dimensional constraints for the tensor coefficients that we mentioned in Section 2.1. Here and in the following we provide the expressions for the matching coefficients in the limit $\epsilon \rightarrow 0$, since the $\mathcal{O}(\epsilon)$ terms are not relevant in two-loop applications.

At NNLO the matching coefficients are no longer given by the finite terms of the respective form factors alone. We now find

$$
\begin{aligned}
C_{S}^{(2)}(u)= & F_{S, 0}^{(2)}(u) \\
& +T_{F} C_{F}\left[\frac{4}{9} \zeta_{3}+\frac{\pi^{2}}{9} g_{0}(u)+\frac{2}{9}\left(6 g_{1}(u)-\pi^{2}\right) L+\left(2 g_{0}(u)+4\right) L^{2}-\frac{14}{9} L^{3}\right] \\
& +C_{F}^{2}\left[g_{3}(u)-g_{0}(u) g_{2}(u)+\left(2 g_{2}(u)-g_{0}(u) g_{1}(u)\right) L\right. \\
& \left.+\frac{1}{2}\left(3 g_{1}(u)-g_{0}(u)^{2}-3 g_{0}(u)\right) L^{2}+\left(\frac{5}{6} g_{0}(u)+2\right) L^{3}-\frac{5}{24} L^{4}\right],
\end{aligned}
$$

and

$$
\begin{aligned}
C_{T}^{1,(2)}(u)= & F_{T, 0}^{1,(2)}(u) \\
& -\frac{1}{2} T_{F} C_{F}\left[\frac{4}{9} \zeta_{3}+\frac{\pi^{2}}{9} g_{0}(u)+\frac{2}{9}\left(6 g_{4}(u)-\pi^{2}\right) L+\left(2 g_{0}(u)-\frac{4}{3}\right) L^{2}-\frac{14}{9} L^{3}\right] \\
& -\frac{1}{2} C_{F}^{2}\left[g_{6}(u)-g_{0}(u) g_{5}(u)+\left(2 g_{5}(u)-g_{0}(u) g_{4}(u)\right) L\right.
\end{aligned}
$$




$$
\left.+\frac{1}{2}\left(3 g_{4}(u)-g_{0}(u)^{2}+g_{0}(u)\right) L^{2}+\left(\frac{5}{6} g_{0}(u)-\frac{2}{3}\right) L^{3}-\frac{5}{24} L^{4}\right]
$$

and

$$
\begin{aligned}
C_{T}^{3,(2)}(u)= & F_{T, 0}^{3,(2)}(u)+T_{F} C_{F}\left[\frac{4}{3} g_{9}(u) L\right] \\
& +C_{F}^{2}\left[g_{11}(u)-g_{0}(u) g_{10}(u)+\left(2 g_{10}(u)-g_{0}(u) g_{9}(u)\right) L+\frac{3}{2} g_{9}(u) L^{2}\right] .
\end{aligned}
$$

The other tensor coefficients are again found to fulfill the four-dimensional constraints

$$
\begin{aligned}
& C_{T}^{2,(2)}(u)=F_{T, 0}^{2,(2)}(u)-C_{F}^{2}\left[g_{0}(u) g_{7}(u)-g_{8}(u)-2 g_{7}(u) L\right]=0, \\
& C_{T}^{4,(2)}(u)=F_{T, 0}^{4,(2)}(u)-C_{F}^{2}\left[g_{0}(u) g_{12}(u)-g_{13}(u)-2 g_{12}(u) L\right]=0,
\end{aligned}
$$

which provides a non-trivial cross check of our calculation.

As a further check of our NNLO results we verified that the matching coefficients obey the renormalization group equation,

$$
\frac{d}{d \ln \mu} C_{i}^{j}(u ; \mu)=\left[\Gamma_{\text {cusp }}\left(\alpha_{s}^{(4)}\right) \ln \frac{u m_{b}}{\mu}+\gamma^{\prime}\left(\alpha_{s}^{(4)}\right)+\gamma_{i}\left(\alpha_{s}^{(5)}\right)\right] C_{i}^{j}(u ; \mu),
$$

which consists of a universal piece related to the renormalization properties of the SCET current with

$$
\Gamma_{\text {cusp }}\left(\alpha_{s}^{(4)}\right)=\sum_{k=1}^{\infty}\left(\frac{\alpha_{s}^{(4)}}{4 \pi}\right)^{k} \Gamma_{\text {cusp }}^{(k)}, \quad \quad \gamma^{\prime}\left(\alpha_{s}^{(4)}\right)=\sum_{k=1}^{\infty}\left(\frac{\alpha_{s}^{(4)}}{4 \pi}\right)^{k} \gamma^{\prime(k)},
$$

and a second term that contains the anomalous dimension of the QCD current with

$$
\gamma_{i}\left(\alpha_{s}^{(5)}\right)=\sum_{k=1}^{\infty}\left(\frac{\alpha_{s}^{(5)}}{4 \pi}\right)^{k} \gamma_{i}^{(k)}
$$

The one- and two-loop coefficients needed for the check read $\Gamma_{\text {cusp }}^{(1)}=4 C_{F}, \gamma^{\prime(1)}=-5 C_{F}$,

$$
\begin{aligned}
\Gamma_{\text {cusp }}^{(2)} & =C_{A} C_{F}\left[\frac{268}{9}-\frac{4 \pi^{2}}{3}\right]-\frac{80}{9} n_{l} T_{F} C_{F} \\
\gamma^{\prime(2)} & =C_{F}^{2}\left[2 \pi^{2}-\frac{3}{2}-24 \zeta_{3}\right]+C_{A} C_{F}\left[22 \zeta_{3}-\frac{1549}{54}-\frac{7 \pi^{2}}{6}\right]+n_{l} T_{F} C_{F}\left[\frac{250}{27}+\frac{2 \pi^{2}}{3}\right]
\end{aligned}
$$

and

$$
\gamma_{S}^{(1)}=6 C_{F}, \quad \gamma_{S}^{(2)}=C_{F}\left[3 C_{F}+\frac{97}{3} C_{A}-\frac{20}{3}\left(n_{l}+1\right) T_{F}\right]
$$




$$
\gamma_{T}^{(1)}=-2 C_{F}, \quad \gamma_{T}^{(2)}=C_{F}\left[19 C_{F}-\frac{257}{9} C_{A}+\frac{52}{9}\left(n_{l}+1\right) T_{F}\right] .
$$

The twofold structure of (39) can be used to distinguish the scale $\mu$, that governs the renormalization group evolution in SCET, from a second scale $\nu$, that is related to the non-conservation of the scalar/tensor current in QCD. More explicitly the distinction between the scales $\mu$ and $\nu$ can be accounted for by writing

$$
C_{i}^{j}(u ; \mu, \nu)=C_{i}^{j}(u ; \mu)+\delta C_{i}^{j}(u ; \mu, \nu),
$$

where the first term on the right-hand side refers to the above expressions for the matching coefficients, $C_{i}^{j}(u ; \mu) \equiv C_{i}^{j}(u)$, while the latter captures the dependence on $\ln (\nu / \mu)$, which vanishes when the two scales are not distinguished. Expanding the new contribution as

$$
\delta C_{i}^{j}=\sum_{k=1}^{\infty}\left(\frac{\alpha_{s}^{(4)}(\mu)}{4 \pi}\right)^{k} \delta C_{i}^{j,(k)}
$$

we find

$$
\delta C_{i}^{j,(1)}(u ; \mu, \nu)=\gamma_{i}^{(1)} C_{i}^{j,(0)} \ln \frac{\nu}{\mu}
$$

in NLO, and

$$
\begin{aligned}
\delta C_{i}^{j,(2)}(u ; \mu, \nu)= & {\left[\frac{\gamma_{i}^{(1)^{2}}}{2}-\gamma_{i}^{(1)} \beta_{0}^{(5)}\right] C_{i}^{j,(0)} \ln ^{2} \frac{\nu}{\mu} } \\
& +\left[\left(\gamma_{i}^{(2)}+\frac{4}{3} T_{F} \gamma_{i}^{(1)} \ln \frac{\mu^{2}}{m_{b}^{2}}\right) C_{i}^{j,(0)}+\gamma_{i}^{(1)} C_{i}^{j,(1)}(u ; \mu)\right] \ln \frac{\nu}{\mu}
\end{aligned}
$$

in NNLO. (Here $\beta_{0}^{(5)}=11 C_{A} / 3-4 / 3 T_{F} n_{f}$ refers to the QCD beta-function with $n_{f}=$ $n_{l}+1$ flavours.) Our final results for the matching coefficients with the two scales $\mu$ and $\nu$ distinct from each other are provided in electronic form in 23 .

The matching coefficients with the SCET and QCD scale distinct from each other can be used for additional cross-checks. The scalar coefficient is not independent but can be related to the vector coefficients by means of the equations of motion, yielding [13]

$$
C_{V}^{1}(u ; \mu)+\left(1-\frac{u}{2}\right) C_{V}^{2}(u ; \mu)+C_{V}^{3}(u ; \mu)=\frac{\bar{m}_{b}(\nu)}{m_{b}} C_{S}(u ; \mu, \nu),
$$

where $\bar{m}_{b}(\nu)$ is the $\overline{\mathrm{MS}}$ renormalized mass in five-flavour QCD. Due to the conservation of the vector current the left-hand side of (48), which happens to be just the coefficient $C_{f_{0}}^{(A 0)}$ from (54) below, is free of $\nu$. Hence the QCD scale must also drop out of the right-hand side. We checked that our results satisfy (48). An equivalent formulation of (48) was given in [9] in terms of a Ward-identity. Also the tensor coefficients at $u=1$, corresponding to $q^{2}=0$, can be checked against existing results in the literature, since they enter the $b \rightarrow s \gamma$ process. From [24] (see also [25]) one can infer the combinations

$$
-2 F_{T}^{1}(u=1)+\frac{1}{2} F_{T}^{2}(u=1)+F_{T}^{3}(u=1)
$$


and

$$
-2 C_{T}^{1}(u=1 ; \mu, \nu)+C_{T}^{3}(u=1 ; \mu, \nu) .
$$

The latter equation can again be checked for distinct $\mu$ and $\nu$, and both (49) and (50)

agree with the formulas in [24]. Note that (50) is just the coefficient $C_{T_{1}}^{(A 0)}$ from (54) at $u=1$.

In Figure 1 we evaluate the matching coefficients for $\mu=\nu=m_{b}$ and $\alpha_{s}^{(4)}\left(m_{b}\right)=0.22$. For completeness we show the full set of matching coefficients $C_{i}^{j}$ that we introduced in Table 1. We see that the NNLO corrections are in general moderate and add in each case constructively to the NLO corrections. In Figure 1 we also show the effect of a finite charm quark mass, which is generally rather small, typically modifying the NNLO correction by about $10-20 \%$.

\section{Exclusive semi-leptonic and radiative $B$ decays}

With the two-loop matching coefficients $C_{i}^{j}$ at hand, we explore several applications to $B$ meson decays in this and the following section. For the numerical study we use the following input parameters: the $b$-quark pole mass $m_{b}=4.8 \mathrm{GeV}$; the renormalization scale of the QCD scalar and tensor currents $\nu=m_{b}$; the hard scale $\mu=m_{b}$. The strong coupling constant is obtained from $\alpha_{s}^{(4)}\left(m_{b}\right)=0.215$ by employing three-loop running $\left(\Lambda_{\overline{\mathrm{MS}}}^{\left(n_{f}=4\right)}=290.9 \mathrm{MeV}\right)$, which gives $\alpha_{s}^{(4)}(1.5 \mathrm{GeV})=0.349$. When we add the hard spectator-scattering contribution from [14] as required for exclusive processes, we need further parameters (such as moments of light-cone distribution amplitudes), for which we use the same values as [14] including the hard-collinear scale $\mu_{\mathrm{hc}}=1.5 \mathrm{GeV}$.

\subsection{Heavy-to-light form factor ratios}

The heavy-to-light form factors in the large-recoil regime, where the light meson momentum is parametrically of order of the heavy-quark mass, take the following factorization formula [4, 7]

$$
F_{i}^{B \rightarrow M}(E)=C_{i}(E) \xi_{a}(E)+\int_{0}^{\infty} \frac{d \omega}{\omega} \int_{0}^{1} d v T_{i}(E ; \ln \omega, v) \phi_{B+}(\omega) \phi_{M}(v),
$$

where $E$ denotes the energy of the light meson $M, \xi_{a}(E)$ is the single non-perturbative form factor (one of two when $M$ is a vector meson), and $\phi_{X}$ the light-cone distribution amplitudes of the $B$ meson and the light meson. The short-distance coefficients $C_{i}$ and the spectator-scattering kernel $T_{i}$ can be calculated in perturbation theory. The two terms in the above equation correspond to the matrix elements of the two terms in the operator matching equation (1). In particular, the two-loop results from the previous section enter the coefficients $C_{i}(E)$ of the first term. The spectator-scattering kernels $T_{i}$ have been calculated at $\mathcal{O}\left(\alpha_{s}\right)$ in [7], and at $\mathcal{O}\left(\alpha_{s}^{2}\right)$ in [13, 14].

In the following we discuss relations between different QCD form factors $F_{i}^{B \rightarrow M}(E)$ that can be deduced from the factorization formula (51). Adopting the same conventions 

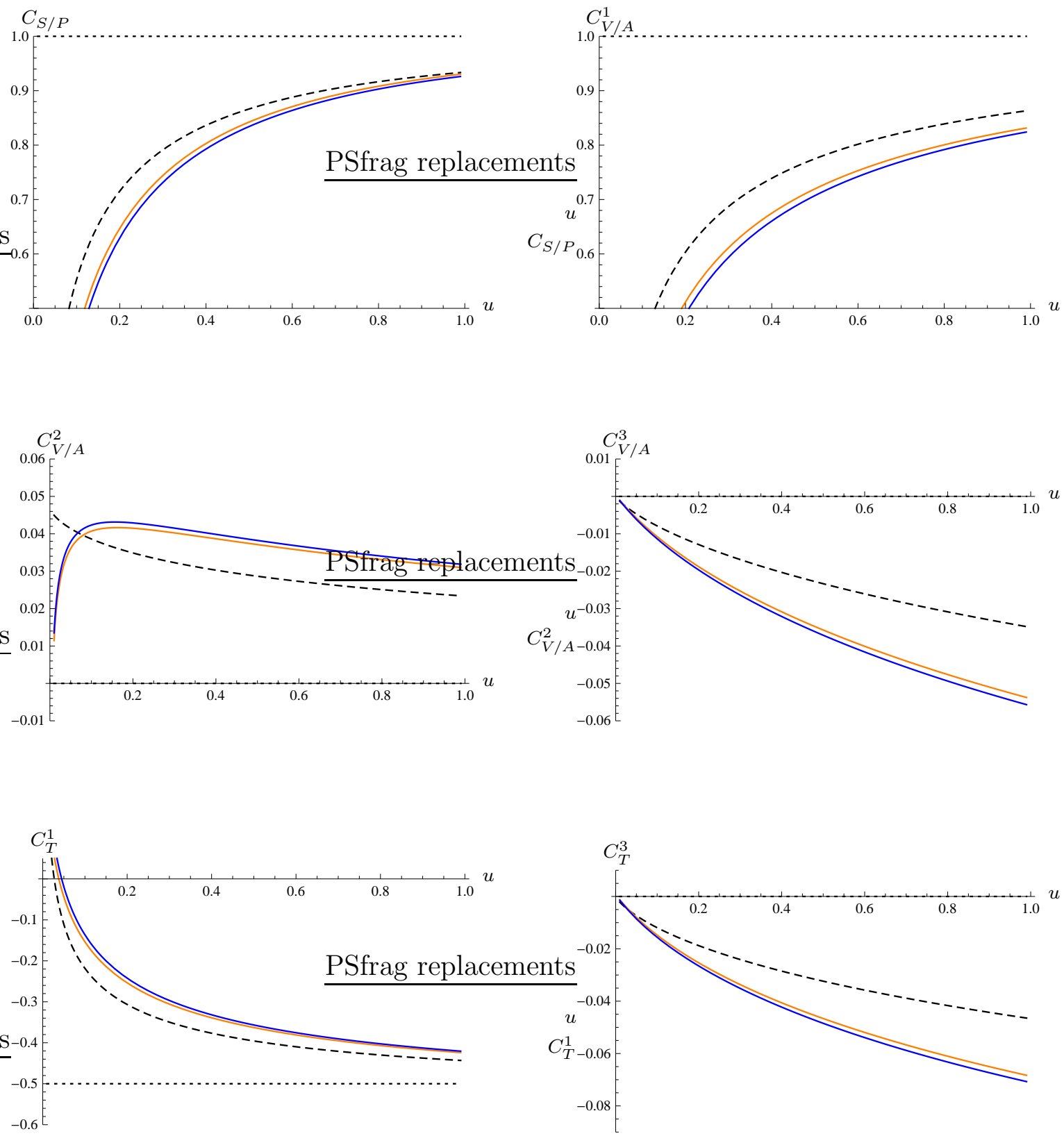

Figure 1: Matching coefficients $C_{i}^{j}$ at the scale $\mu=\nu=m_{b}$ as a function of $u$ (the momentum transfer is given by $\left.q^{2}=(1-u) m_{b}^{2}\right)$. The dotted horizontal lines show the tree level results, the dashed lines the one-loop approximation and the solid lines the two-loop approximation with massless charm quarks (orange/light grey) and massive charm quarks with $m_{c} / m_{b}=0.3$ (blue/dark grey).

and notations as [14], we can express the three independent $B \rightarrow P$ form factors as

$$
\begin{aligned}
f_{+}(E) & =C_{f_{+}}^{(A 0)}(E) \xi_{P}(E)+\int d \tau C_{f_{+}}^{(B 1)}(E, \tau) \Xi_{P}(\tau, E), \\
\frac{m_{B}}{2 E} f_{0}(E) & =C_{f_{0}}^{(A 0)}(E) \xi_{P}(E)+\int d \tau C_{f_{0}}^{(B 1)}(E, \tau) \Xi_{P}(\tau, E),
\end{aligned}
$$




$$
\frac{m_{B}}{m_{B}+m_{P}} f_{T}(E)=C_{f_{T}}^{(A 0)}(E) \xi_{P}(E)+\int d \tau C_{f_{T}}^{(B 1)}(E, \tau) \Xi_{P}(\tau, E),
$$

and the seven independent $B \rightarrow V$ form factors as

$$
\begin{aligned}
\frac{m_{B}}{m_{B}+m_{V}} V(E) & =C_{V}^{(A 0)}(E) \xi_{\perp}(E)+\int d \tau C_{V}^{(B 1)}(E, \tau) \Xi_{\perp}(\tau, E), \\
\frac{m_{V}}{E} A_{0}(E) & =C_{f_{0}}^{(A 0)}(E) \xi_{\|}(E)+\int d \tau C_{f_{0}}^{(B 1)}(E, \tau) \Xi_{\|}(\tau, E), \\
\frac{m_{B}+m_{V}}{2 E} A_{1}(E) & =C_{V}^{(A 0)}(E) \xi_{\perp}(E)+\int d \tau C_{V}^{(B 1)}(E, \tau) \Xi_{\perp}(\tau, E), \\
\frac{m_{B}+m_{V}}{2 E} A_{1}(E) & -\frac{m_{B}-m_{V}}{m_{B}} A_{2}(E) \\
T_{1}(E) & =C_{f_{+}}^{(A 0)}(E) \xi_{\|}(E)+\int d \tau C_{f_{+}}^{(B 1)}(E, \tau) \Xi_{\|}(\tau, E), \\
\frac{m_{B}}{2 E} T_{2}(E) & =C_{T_{1}}^{(A 0)}(E) \xi_{\perp}(E)+\int d \tau C_{T_{1}}^{(B 1)}(E, \tau) \Xi_{\perp}(\tau, E), \\
\frac{m_{B}}{2 E} T_{2}(E)-T_{3}(E) & =C_{f_{T}}^{(A 0)}(E) \xi_{\|}(E)+\int d \tau C_{f_{T}}^{(B 1)}(E, \tau) \Xi_{\|}(\tau, E) .
\end{aligned}
$$

Here $m_{B}$ represents the $B$ meson mass, $m_{P}$ and $m_{V}$ refer to the pseudoscalar and vector light meson masses, respectively. The coefficient functions $C_{F}^{(A 0)}$ and $C_{F}^{(B 1)}$ are defined as linear combinations of the matching coefficients of two- ("A0-type") and three-body ("Btype") SCET operators, while $\Xi_{a}(\tau, E)$ denotes the matrix elements of the three-body operators $O_{i}^{(B 1) j \mu}\left(s_{1}, s_{2}\right)$, see (11). In terms of the coefficients $C_{i}^{j}$ introduced in previous sections, the five independent A0-coefficients are given by

$$
\begin{aligned}
& C_{f_{+}}^{(A 0)}=C_{V}^{1}(u ; \mu)+\frac{u}{2} C_{V}^{2}(u ; \mu)+C_{V}^{3}(u ; \mu), \\
& C_{f_{0}}^{(A 0)}=C_{V}^{1}(u ; \mu)+\left(1-\frac{u}{2}\right) C_{V}^{2}(u ; \mu)+C_{V}^{3}(u ; \mu), \\
& C_{f_{T}}^{(A 0)}=-2 C_{T}^{1}(u ; \mu, \nu)+C_{T}^{2}(u ; \mu, \nu)-C_{T}^{4}(u ; \mu, \nu), \\
& C_{V}^{(A 0)}=C_{V}^{1}(u ; \mu) \\
& C_{T_{1}}^{(A 0)}=-2 C_{T}^{1}(u ; \mu, \nu)+\left(1-\frac{u}{2}\right) C_{T}^{2}(u ; \mu, \nu)+C_{T}^{3}(u ; \mu, \nu) .
\end{aligned}
$$

Recall that in $D=4$ dimensions one has $C_{T}^{2}=C_{T}^{4}=0$. The variable $E$ used in (52) and (53) is related to $u$ through $u=2 E / m_{B}$. The five independent B-coefficients are given in Appendix A2 of [14].

From (52) and (53), we have the following two identities

$$
\frac{m_{B}}{m_{B}+m_{V}} V(E)=\frac{m_{B}+m_{V}}{2 E} A_{1}(E), \quad T_{1}(E)=\frac{m_{B}}{2 E} T_{2}(E)
$$


up to power corrections [26]. In the physical form factor scheme [7, 14], where the $\mathrm{SCET}_{\mathrm{I}}$ form factors $\xi_{a}(E)$ are defined in terms of three QCD form factors,

$$
\xi_{P}^{\mathrm{FF}} \equiv f_{+}, \quad \xi_{\perp}^{\mathrm{FF}} \equiv \frac{m_{B}}{m_{B}+m_{V}} V, \quad \xi_{\|}^{\mathrm{FF}} \equiv \frac{m_{B}+m_{V}}{2 E} A_{1}-\frac{m_{B}-m_{V}}{m_{B}} A_{2},
$$

the five remaining form factors read

$$
\begin{aligned}
\frac{m_{B}}{2 E} f_{0} & =R_{0} \xi_{P}^{\mathrm{FF}}+\left(C_{f_{0}}^{(B 1)}-C_{f_{+}}^{(B 1)} R_{0}\right) \star \Xi_{P}, \\
\frac{m_{B}}{m_{B}+m_{P}} f_{T} & =R_{T} \xi_{P}^{\mathrm{FF}}+\left(C_{f_{T}}^{(B 1)}-C_{f_{+}}^{(B 1)} R_{T}\right) \star \Xi_{P}, \\
T_{1} & =R_{\perp} \xi_{\perp}^{\mathrm{FF}}+\left(C_{T_{1}}^{(B 1)}-C_{V}^{(B 1)} R_{\perp}\right) \star \Xi_{\perp}, \\
\frac{m_{V}}{E} A_{0} & =R_{0} \xi_{\|}^{\mathrm{FF}}+\left(C_{f_{0}}^{(B 1)}-C_{f_{+}}^{(B 1)} R_{0}\right) \star \Xi_{\|}, \\
\frac{m_{B}}{2 E} T_{2}-T_{3} & =R_{T} \xi_{\|}^{\mathrm{FF}}+\left(C_{f_{T}}^{(B 1)}-C_{f_{+}}^{(B 1)} R_{T}\right) \star \Xi_{\|} .
\end{aligned}
$$

In this scheme there are only three non-trivial ratios $R$ and three non-trivial combinations of B-coefficients, defined, respectively, as

$$
\begin{aligned}
R_{0}(u) & \equiv \frac{C_{f_{0}}^{(A 0)}}{C_{f_{+}}^{(A 0)}}=1+\frac{\alpha_{s}^{(4)}}{4 \pi} C_{F}\left[2+g_{9}(u)\right]\left[1+\frac{\alpha_{s}^{(4)}}{4 \pi} \beta_{0}^{(4)} L_{\mu}\right] \\
+ & \left(\frac{\alpha_{s}^{(4)}}{4 \pi}\right)^{2}\left\{C_{F}^{2} j_{1}(u)+C_{F} C_{A} j_{2}(u)+C_{F} n_{l} T_{F} j_{3}(u)+C_{F} T_{F} j_{4}(u)\right\}+\mathcal{O}\left(\alpha_{s}^{3}\right) \\
R_{T}(u) & \equiv \frac{C_{f_{T}}^{(A 0)}}{C_{f_{+}}^{(A 0)}}=1+\frac{\alpha_{s}^{(4)}}{4 \pi} C_{F}\left[-L_{\nu}-g_{9}(u)\right]\left[1+\frac{\alpha_{s}^{(4)}}{4 \pi} \beta_{0}^{(4)} L_{\mu}\right] \\
+ & \left(\frac{\alpha_{s}^{(4)}}{4 \pi}\right)^{2}\left\{C_{F}^{2}\left[\frac{L_{\nu}^{2}}{2}+\left(\frac{19}{2}+g_{9}(u)\right) L_{\nu}+j_{5}(u)\right]+C_{F} C_{A}\left[\frac{11 L_{\nu}^{2}}{6}-\frac{257 L_{\nu}}{18}+j_{6}(u)\right]\right. \\
+ & \left.C_{F} n_{l} T_{F}\left[-\frac{2 L_{\nu}^{2}}{3}+\frac{26 L_{\nu}}{9}+j_{7}(u)\right]+C_{F} T_{F}\left[-\frac{2 L_{\nu}^{2}}{3}+\frac{26 L_{\nu}}{9}+j_{8}(u)\right]\right\}+\mathcal{O}\left(\alpha_{s}^{3}\right) \\
R_{\perp}(u) & \equiv \frac{C_{T_{1}}^{(A 0)}}{C_{V}^{(A 0)}}=1+\frac{\alpha_{s}^{(4)}}{4 \pi} C_{F}\left[-L_{\nu}+\frac{1}{2} g_{9}(u)\right]\left[1+\frac{\alpha_{s}^{(4)}}{4 \pi} \beta_{0}^{(4)} L_{\mu}\right] \\
+ & \left(\frac{\alpha_{s}^{(4)}}{4 \pi}\right)^{2}\left\{C_{F}^{2}\left[\frac{L_{\nu}^{2}}{2}+\left(\frac{19}{2}-\frac{1}{2} g_{9}(u)\right) L_{\nu}-\frac{1}{2} j_{5}(u)+j_{9}(u)\right]\right. \\
+ & C_{F} C_{A}\left[\frac{11 L_{\nu}^{2}}{6}-\frac{257 L_{\nu}}{18}-\frac{1}{2} j_{6}(u)+j_{10}(u)\right]+C_{F} n_{l} T_{F}\left[-\frac{2 L_{\nu}^{2}}{3}+\frac{26 L_{\nu}}{9}-\frac{1}{2} j_{7}(u)\right.
\end{aligned}
$$




$$
\left.\left.+\frac{2 \pi^{2}}{3}+\frac{205}{36}\right]+C_{F} T_{F}\left[-\frac{2 L_{\nu}^{2}}{3}+\frac{26 L_{\nu}}{9}-\frac{1}{2} j_{8}(u)-\frac{4 \pi^{2}}{3}+\frac{421}{36}\right]\right\}+\mathcal{O}\left(\alpha_{s}^{3}\right)
$$

and

$$
\begin{aligned}
& C_{0+}^{(B 1)}(\tau, E)=C_{f_{0}}^{(B 1)}(\tau, E)-C_{f_{+}}^{(B 1)}(\tau, E) R_{0}(E), \\
& C_{T+}^{(B 1)}(\tau, E)=C_{f_{T}}^{(B 1)}(\tau, E)-C_{f_{+}}^{(B 1)}(\tau, E) R_{T}(E), \\
& C_{T_{1} V}^{(B 1)}(\tau, E)=C_{T_{1}}^{(B 1)}(\tau, E)-C_{V}^{(B 1)}(\tau, E) R_{\perp}(E) .
\end{aligned}
$$

We denote $L_{\mu}=\ln \left(\mu^{2} / m_{b}^{2}\right), L_{\nu}=\ln \left(\nu^{2} / m_{b}^{2}\right)$, and $\beta_{0}^{(4)}=11 / 3 C_{A}-4 / 3 T_{F} n_{l}$. The functions $j_{i}(u)$ can be found in Appendix $\mathrm{B}$. One recognizes the relatively simple structure of the ratios $R_{X}$ in the physical form factor scheme. Compared to the matching coefficients, where we encounter up to the fourth power of logarithms, the ratios $R_{X}$ have logarithmic dependences that are at most quadratic, since the universal Sudakov logarithms cancel in the ratios.

As expected in any perturbative QCD calculation, the higher-order correction is necessary to eliminate scale ambiguities. While the A0-coefficients $C_{X}^{(A 0)}$ depend on the hard scale $\mu$ (which is cancelled by the corresponding dependence of the $\mathrm{SCET}_{\mathrm{I}}$ form factors $\left.\xi_{a}(E)\right)$, the $\mu$ dependence of the ratios $R_{X}(X=0, T, \perp)$ arises only from the scale-dependence of $\alpha_{s}(\mu)$ and should be reduced after including the higher-order correction. In Figure 2, we show the dependence of the three ratios $R_{X}$ on the scale $\mu$ at $u=0.85$ (corresponding to the light-meson energy $E=u m_{B} / 2=2.24 \mathrm{GeV}$ or momentum transfer $q^{2}=4.18 \mathrm{GeV}^{2}$ ) and fixed renormalization scale $\nu=m_{b}$ of the QCD tensor current. In the absence of radiative and power corrections, all these coefficients equal 1 (dotted lines). We observe that the scale dependence is reduced at the two-loop order for the ratios $R_{0, T}$, but not for $R_{\perp}$, which receives a large two-loop correction.

Since the A0-type coefficients $C_{X}^{(A 0)}$ and hence the ratios $R_{X}$ also depend on the momentum transfer $q^{2}$, we show in Figure 3 these coefficients as a function of $u$ (related to light-meson energy $E=u m_{B} / 2$ or momentum transfer $\left.q^{2}=(1-u) m_{B}^{2}\right)$, with the scales fixed at $\nu=\mu=m_{b}$. As illustrated in Figure 3, the NNLO correction to all the five coefficients $C_{X}^{(A 0)}$ is quite similar and adds in each case constructively to the NLO result; among the three ratios $R_{X}$, the two-loop correction to $R_{\perp}$, i.e. to the ratio of the tensor and vector form factor, $T_{1} / V$, is most significant.

To further investigate these two-loop corrections to the form factor ratios, following [14] we also take the $B \rightarrow \pi$ and $B \rightarrow \rho$ transitions as examples. Seven ratios among the total of ten pion and $\rho$ meson form factors can be obtained from the two identities (55), which do not receive any perturbative corrections, and the five relations that follow from (57) by dividing through the appropriate $\xi_{a}^{\mathrm{FF}}$. The $q^{2}$ dependence of these form factor ratios are shown in Figure 4. As in [14] the $q^{2}$-dependence of the $\xi_{a}^{\mathrm{FF}}$ in the normalization of the spectator-scattering correction is taken from the QCD sum rule calculation. The ratios are normalized such that in absence of any radiative and power corrections they equal 1 for all $q^{2}$. Our final results, including both $R_{X}$ and the spectator-scattering term to order $\alpha_{s}^{2}$, are shown as solid dark grey (blue in colour) curves, while the results with 

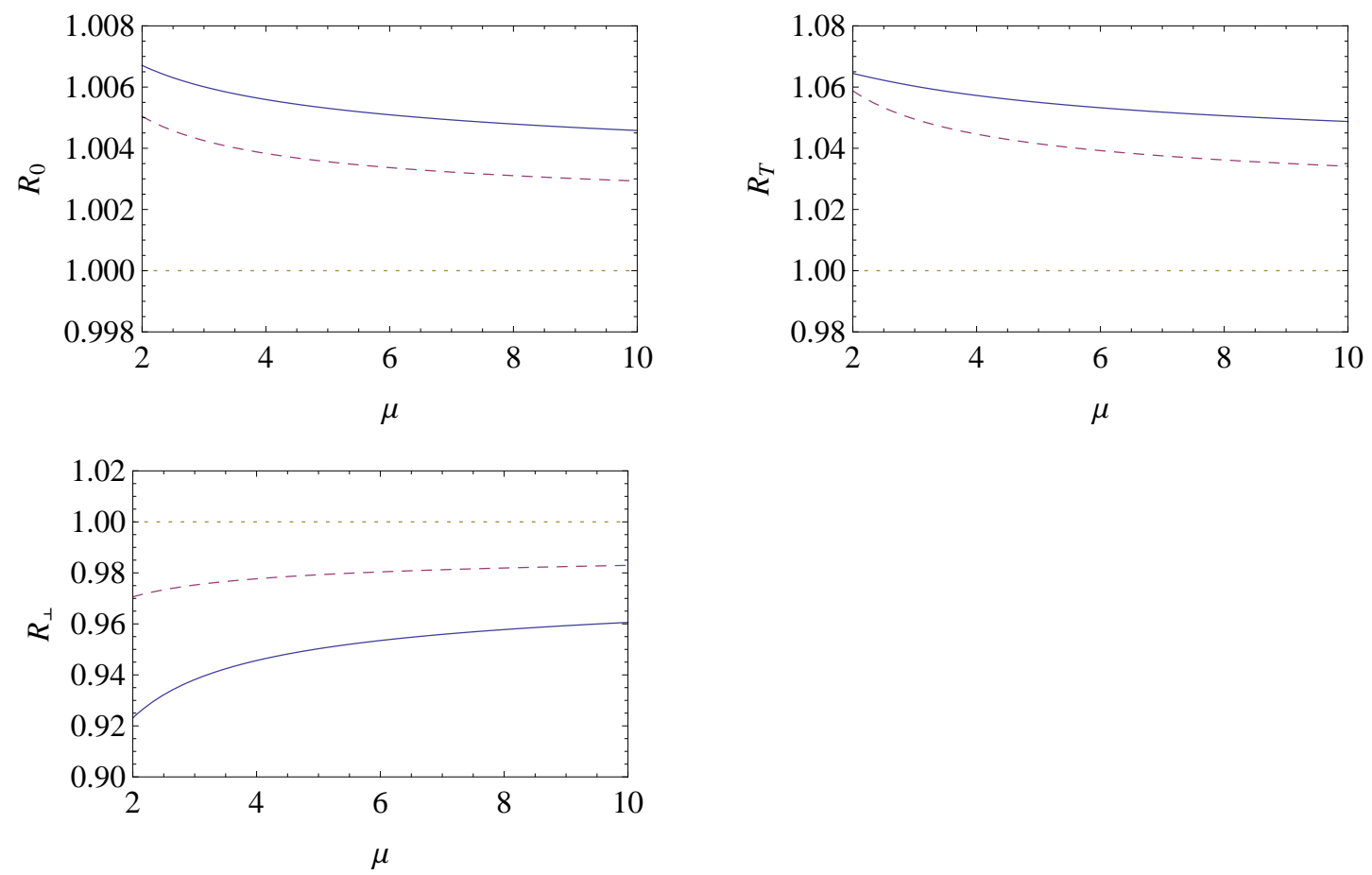

Figure 2: Dependence of the ratios $R_{X}(X=0, T, \perp)$ defined in (58) on the scale $\mu$, with $u=0.85$ (corresponding to the light-meson energy $E=u m_{B} / 2=2.24 \mathrm{GeV}$ or momentum transfer $q^{2}=4.18 \mathrm{GeV}^{2}$ ) and $\nu=m_{b}$ (the renormalization scale of the QCD tensor current). All of them equal 1 in the absence of radiative and power corrections (dotted line). The solid and dashed lines denote the NNLO and NLO results, respectively.

$R_{X}$ evaluated only at NLO as solid light grey (orange in colour) ones. One can see that the radiative correction always enhances the symmetry-breaking effect, and the NNLO term is generally quite moderate; the most significant effect from the two-loop correction is on the ratio $T_{1} / V$ (through the ratio $R_{\perp}$ ). To see the relative size of the two terms in the factorization formula (51), we also show the result without the spectator-scattering term (dashed curves with blue/dark grey and orange/light grey denoting the NNLO and NLO results, respectively). Comparing the solid with the dashed curves, one can see that the radiative correction from the A0-coefficients $C_{X}^{(A 0)}$ is always smaller than the spectator-scattering contribution.

To compare our results with the QCD sum rule calculations [27], the sum rule predictions for these form factor ratios are shown as dash-dotted curves in Figure 4. One notices that, while the sum rule calculation generally satisfies the symmetry relations better than predicted on the basis of the heavy-quark limit corrected by radiative and spectator-scattering effects, see for instance the lower right panel of Figure 4, there are also significant differences concerning the sign of the correction, which might be due to $1 / m_{b}$ power corrections or ununderstood systematics of the sum rule calculations; further detailed discussions could be found in [7, 14, 28]. The new two-loop correction does not affect the conclusions on this point. 

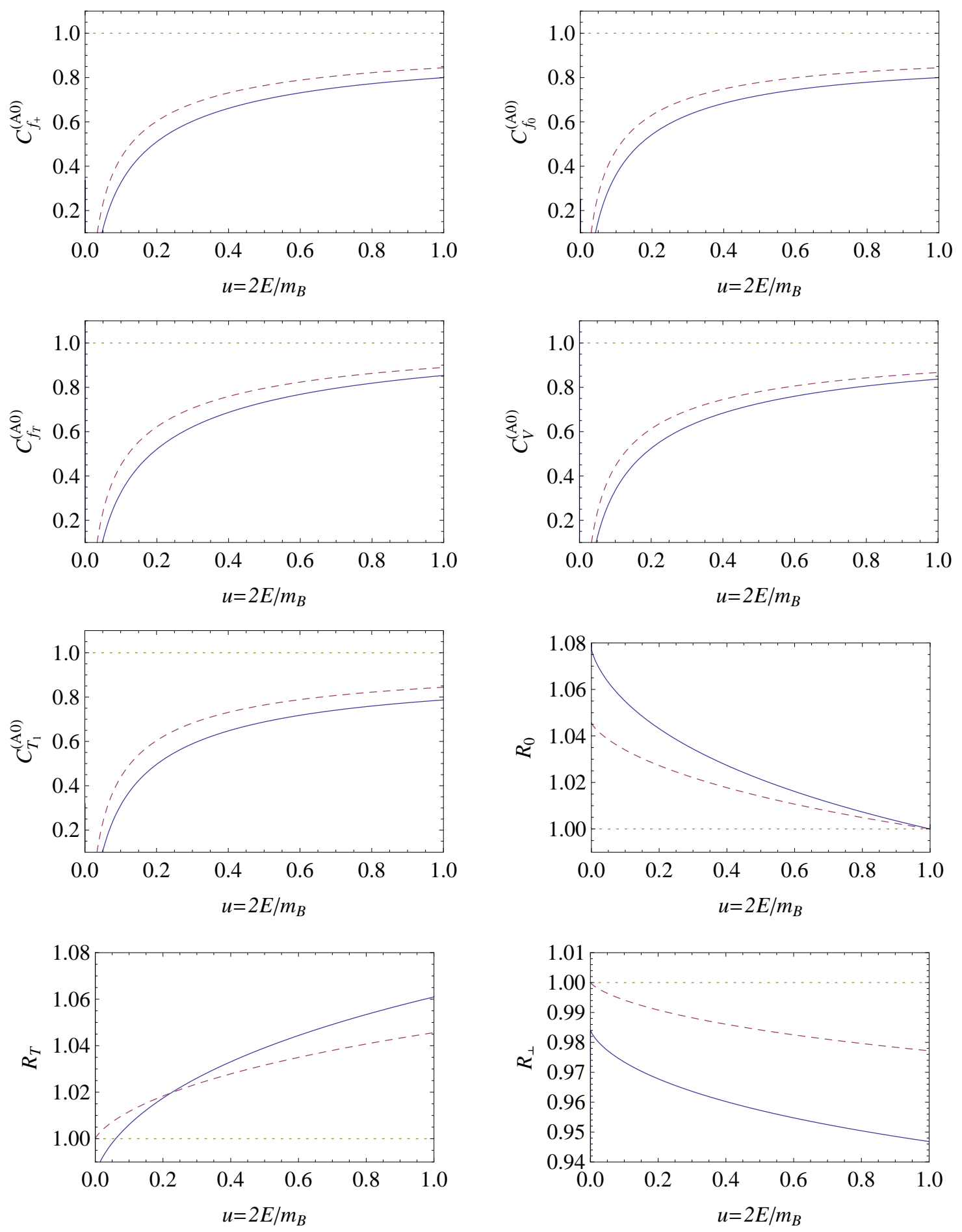

Figure 3: The A0-type coefficients $C_{X}^{(A 0)}$ and the ratios $R_{X}(X=0, T, \perp)$ defined in (58) as a function of $u$ (related to light-meson energy $E=u m_{B} / 2$ or momentum transfer $q^{2}=$ $\left.(1-u) m_{B}^{2}\right)$, with the scales fixed at $\nu=\mu=m_{b}$. The legend is the same as in Figure Q. 

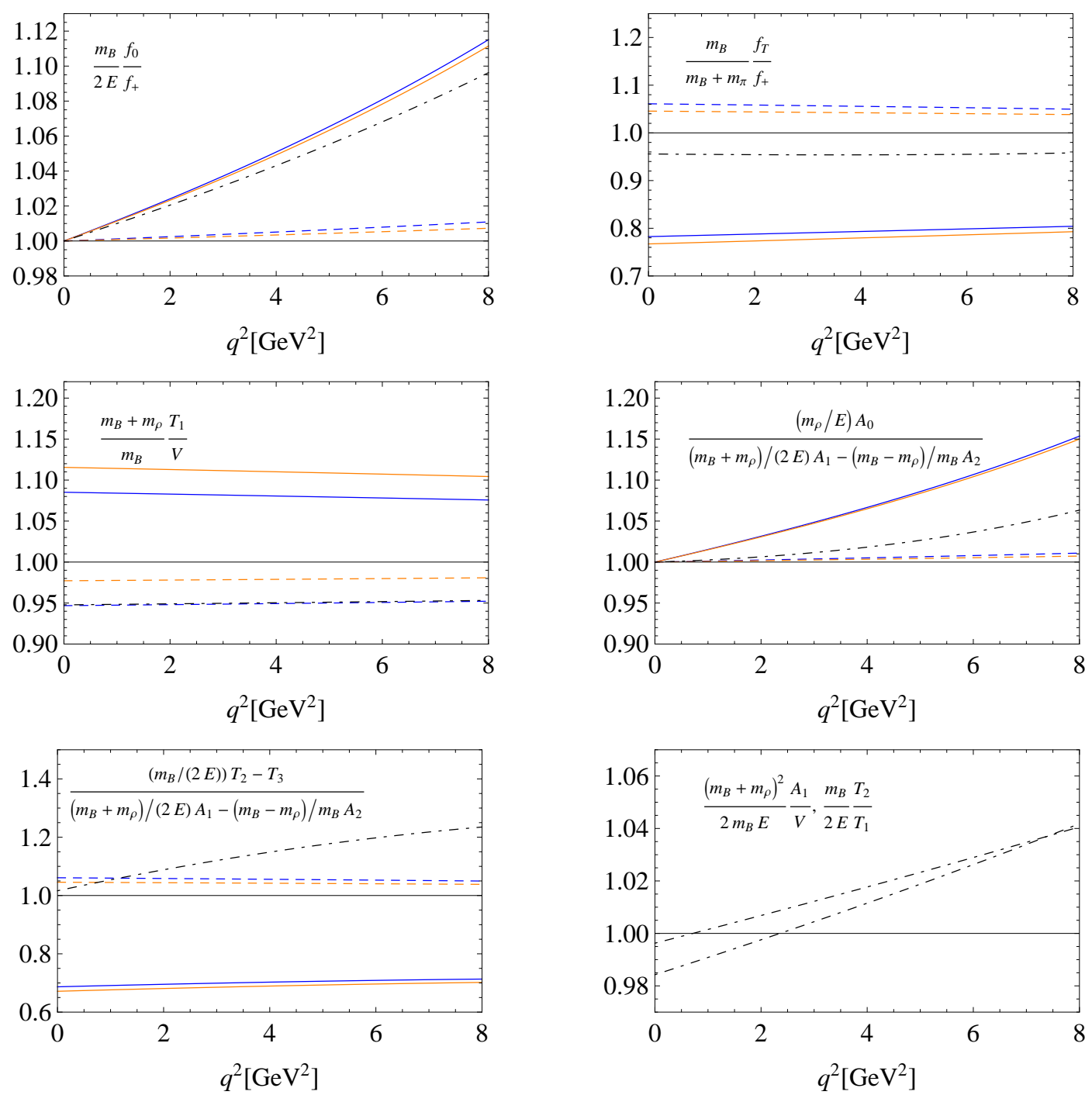

Figure 4: Corrections to the $B \rightarrow \pi$ and $B \rightarrow \rho$ form factor ratios as a function of momentum transfer $q^{2}$. All the ratios equal 1 in the absence of radiative corrections. Solid curves: full results with $R_{X}$ evaluated at $N N L O$ (blue/dark grey) and $N L O$ (orange/light grey), including the spectator-scattering term; Dashed: results without the spectator-scattering contribution; Dashdotted: results from QCD sum rule calculation. The lower right panel shows the two form factor ratios that equal 1 at leading power. For comparison, the QCD sum rule results for these two ratios are also shown (upper line refers to $A_{1} / V$, lower line to $T_{2} / T_{1}$ ).

\subsection{Exclusive radiative $B$ decays}

As factorization calculations of exclusive radiative and hadronic $B$ decays involving only light mesons make use of the heavy-to-light form factors at maximal recoil, it is of interest to investigate the short-distance corrections at $u=1$, i.e. $E=m_{B} / 2$ or $q^{2}=0$. In this 
subsection we shall consider the following two ratios [14]

$$
\begin{aligned}
\mathcal{R}_{1}(E) & \equiv \frac{m_{B}}{m_{B}+m_{P}} \frac{f_{T}(E)}{f_{+}(E)}=R_{T}(E)+\int_{0}^{1} d \tau C_{T+}^{(B 1)}(\tau, E) \frac{\Xi_{P}(\tau, E)}{f_{+}(E)} \\
\mathcal{R}_{2}(E) & \equiv \frac{m_{B}+m_{V}}{m_{B}} \frac{T_{1}(E)}{V(E)}=R_{\perp}(E)+\frac{m_{B}+m_{V}}{m_{B}} \int_{0}^{1} d \tau C_{T_{1} V}^{(B 1)}(\tau, E) \frac{\Xi_{\perp}(\tau, E)}{V(E)},
\end{aligned}
$$

defined in the physical form factor scheme.

At $u=1$ and assuming the asymptotic form for the light-meson distribution amplitude $\phi_{M}(v)=6 v \bar{v}$, the analytic expressions for these two ratios simplify considerably, even at NNLO. As the spectator-scattering contribution is already given by Eq. (124) in [14], here we give only the expressions for the ratios $R_{T, \perp}$ at $u=1$ (as a consequence of the equations of motion, we have $\left.R_{0}(u=1) \equiv 1\right)$,

$$
\begin{aligned}
R_{T}(u=1)= & 1+\frac{\alpha_{s}^{(4)}}{4 \pi}\left[\frac{8}{3}-\frac{4}{3} L_{\nu}\right]+\left(\frac{\alpha_{s}^{(4)}}{4 \pi}\right)^{2}\left[-\frac{100}{9} L_{\mu} L_{\nu}+\frac{200}{9} L_{\mu}+6 L_{\nu}^{2}-\frac{922}{27} L_{\nu}\right. \\
& \left.-\frac{16}{3} \zeta(3)+\frac{10}{3} \pi^{4}-\frac{952}{27} \pi^{2}+\frac{8047}{162}+\frac{128}{27} \pi^{2} \ln 2\right], \\
R_{\perp}(u=1)= & 1+\frac{\alpha_{s}^{(4)}}{4 \pi}\left[-\frac{4}{3}-\frac{4}{3} L_{\nu}\right]+\left(\frac{\alpha_{s}^{(4)}}{4 \pi}\right)^{2}\left[-\frac{100}{9} L_{\mu} L_{\nu}-\frac{100}{9} L_{\mu}+6 L_{\nu}^{2}-\frac{778}{27} L_{\nu}\right. \\
& \left.+4 \zeta(3)-\frac{5}{3} \pi^{4}+\frac{428}{27} \pi^{2}-\frac{13013}{162}-\frac{88}{27} \pi^{2} \ln 2\right],
\end{aligned}
$$

with $L_{\mu}=\ln \left(\mu^{2} / m_{b}^{2}\right), L_{\nu}=\ln \left(\nu^{2} / m_{b}^{2}\right)$, and $n_{l}=4$ has been used. Using the three-loop running coupling and specifying to the pion $\left(\mathcal{R}_{1}\right)$ and $\rho$ meson $\left(\mathcal{R}_{2}\right)$, numerically we obtain (setting $\nu=\mu=m_{b}$ )

$$
\begin{aligned}
\mathcal{R}_{1}\left(E_{\max }\right)= & 1+[0.046(\mathrm{NLO})+0.015(\mathrm{NNLO})]\left(R_{T}\right) \\
& -0.160\left\{1+0.524(\mathrm{NLO} \text { spec. })-0.002\left(\delta_{\log }^{\|}\right)\right\} \\
= & 0.817, \\
\mathcal{R}_{2}\left(E_{\max }\right)= & 1-[0.023(\mathrm{NLO})+0.030(\mathrm{NNLO})]\left(R_{\perp}\right) \\
& +0.084\left\{1+0.406(\mathrm{NLO} \text { spec. })+0.032\left(\delta_{\log }^{\|}\right)\right\} \\
= & 1.067 .
\end{aligned}
$$

In these expressions we separated the symmetry-conserving (first number, normalized to 1), A0- and B-type corrections (denoted by $R_{T, \perp}$ and the remaining terms, respectively). The parameter $\delta_{\log }^{\|}$denotes the small effect from renormalization-group summation and has the same meaning as in Eq. (124) of [14]. We observe that the A0-type and 
spectator-scattering corrections always have opposite sign, but the latter are larger and determine the sign of the deviation from the symmetry limit. We also notice that the two-loop correction to $R_{\perp}$ is more significant than to $R_{T}$. The small numerical difference of spectator-scattering contribution relative to Eq. (124) in [14] is due to the fact that now the 3-loop running coupling is used. For comparison the QCD sum rule calculation [27] gives $\mathcal{R}_{1}=0.955$ and $\mathcal{R}_{2}=0.947$. For the tensor-to-vector ratio $\mathcal{R}_{2}$, one notices that the sign of the symmetry-breaking correction between these two methods is opposite. Since the form factor ratio $T_{1} / V$ is important for radiative and electroweak penguin decays (see the discussion in Section 5.2 of [14]), the discrepancy between the SCET and QCD sum rules results for $\mathcal{R}_{2}$ suggests that a dedicated analysis of symmetry breaking corrections to form factors (rather than the form factors themselves) with the QCD sum rule method should be performed.

\section{Semi-inclusive $\bar{B} \rightarrow X_{s} \ell^{+} \ell^{-}$decays}

Rare inclusive $B$-meson decays induced by the quark level transition $b \rightarrow s \ell^{+} \ell^{-}$are highly sensitive to new physics. Due to the presence of two extra operators $(\bar{\ell} \ell)_{V, A}(\bar{s} b)_{V-A}$ in the effective Hamiltonian and the availability of additional kinematical observables, such as the dilepton invariant mass $\left(q^{2}\right)$ spectrum and the forward-backward asymmetry, the $b \rightarrow s \ell^{+} \ell^{-}$decay provides complementary information relative to the radiative $b \rightarrow s \gamma$ process.

The exclusive decay process $B \rightarrow K^{*} \ell^{+} \ell^{-}$has been studied in great detail, both with respect to its QCD dynamics [29] and to the sensitivity of various observables to new physics [30], because it can be measured relatively easily at hadron colliders. Also on the inclusive decay process $\bar{B} \rightarrow X_{s} \ell^{+} \ell^{-}$dedicated work exists on higher order radiative corrections (see [31] for recent reviews), power corrections [32,33], and on the identification of additional kinematic observables [34].

The low dilepton invariant mass region, $1 \mathrm{GeV}^{2} \leq q^{2} \leq 6 \mathrm{GeV}^{2}$ is particularly interesting, since it benefits from smaller theoretical uncertainties and a higher rate. At somewhat higher $q^{2}$ the spectrum is dominated by charmonium resonances (which also determine the integrated decay rate, see the discussion in [35]). On the other hand, for $q^{2}<1 \mathrm{GeV}^{2}$, the branching ratio is determined largely by the contribution from almost real intermediate photons, and hence contains essentially the same information as the $b \rightarrow s \gamma$ transition.

In the following we discuss semi-inclusive $\bar{B} \rightarrow X_{s} \ell^{+} \ell^{-}$decay, where the hadronic final state $X_{s}$ is constrained to have small invariant mass $m_{X}$ and $q^{2}$ is in the range from $1 \mathrm{GeV}^{2}$ to $6 \mathrm{GeV}^{2}$. In this kinematic region (the so-called "shape function region"), the outgoing hadronic state is jet-like and the relevant degrees of freedom are hardcollinear and soft modes. The semi-inclusive decay rates can be calculated by matching the effective weak interaction Hamiltonian to soft-collinear effective theory. At the leading

order in the $\Lambda_{\mathrm{QCD}} / m_{b}$ expansion, the decay rates can be factorized into process-dependent hard functions $h^{[0]}$, related to physics at the hard scale $\mu \sim m_{b}$ and above, a universal jet 
function $J$, related to physics at the intermediate hard-collinear scale $\mu_{\mathrm{hc}} \sim \sqrt{m_{b} \Lambda_{\mathrm{QCD}}}$, as well as a universal non-perturbative shape function $S$, describing the internal soft dynamics of the $B$ meson, with the following schematic form [36, 37]

$$
\mathrm{d} \Gamma^{[0]}=h^{[0]} \times J \otimes S,
$$

a result already applied extensively to inclusive $\bar{B} \rightarrow X_{u} \ell \bar{\nu}$ and $\bar{B} \rightarrow X_{s} \gamma$ decays in the shape-function region. The two-loop matching coefficients of the tensor currents calculated in the present paper provide further input to reaching NNLO $\left(\alpha_{s}^{2}\right)$ accuracy in $h^{[0]}$ and the entire differential decay rate $\mathrm{d} \Gamma^{[0]}$. Compared to exclusive decays mediated by the $b \rightarrow s \ell^{+} \ell^{-}$transition [29] the semi-inclusive case has the advantage that the theoretically less certain spectator-scattering contributions to the currents that enter the exclusive form factors are power-suppressed and can be dropped.

In the following we will be mainly interested in the forward-backward asymmetry of the differential rate integrated up to an invariant mass $m_{X}^{\text {cut }}$ in the final state. We briefly review the theoretical description of this quantity, adopting the same conventions and notation as [37, to which we also refer for further details. The short-distance coefficients $h^{[0]}$ at the hard matching scale $\mu$ are composed of products of two factors, since the hadronic part of the effective weak interaction Hamiltonian is first matched to two QCD (rather than SCET) currents,

$$
J_{9}^{\mu}=\bar{s} \gamma^{\mu} P_{L} b, \quad J_{7}^{\mu}=\left.\frac{2 m_{b}}{q^{2}} \bar{s} i q_{\rho} \sigma^{\rho \mu} P_{R} b\right|_{\nu=m_{b}},
$$

with coefficients $C_{i}^{\text {incl }}\left(q^{2}, \mu\right)$ and $P_{L, R}=\left(1 \mp \gamma_{5}\right) / 2$. Moreover, $m_{b}$ in $J_{7}^{\mu}$ refers to the bottom quark pole mass. The QCD currents are then related to the corresponding SCET currents,

$$
\begin{aligned}
J_{9}^{\mu} & =\sum_{i=1,2,3} c_{i}^{9}(u, \mu)\left[\bar{\xi} W_{h c}\right] \Gamma_{9, i}^{\mu} h_{v}, \\
J_{7}^{\mu} & =\frac{2 m_{b}}{q^{2}} \sum_{i=1,2} c_{i}^{7}(u, \mu)\left[\bar{\xi} W_{h c}\right] \Gamma_{7, i}^{\mu} h_{v} .
\end{aligned}
$$

These equations represent the momentum space versions of (1). The variable $u$ is related to the kinematics of the process by $u=p^{-} / m_{b}$, where

$$
p^{-}=n_{+} p=m_{b}-\frac{q^{2}}{m_{B}-p_{X}^{+}},
$$

and $p_{X}^{+}=n_{-} p_{X} \ll m_{B}$ is the small light-cone component of the hadronic final state's momentum. The basis of Dirac structures is chosen as

$$
\begin{aligned}
\Gamma_{9, i}^{\mu} & =P_{R}\left\{\gamma^{\mu}, v^{\mu}, q^{\mu}\right\} \\
\Gamma_{7, i}^{\mu} & =P_{R}\left\{i q_{\nu} \sigma^{\nu \mu}, q_{\nu}\left(q^{\nu} v^{\mu}-q^{\mu} v^{\nu}\right)\right\}
\end{aligned}
$$


As noted in [37], the choice of $q^{\mu}$ instead of $n_{-}^{\mu}$ for $\Gamma_{9,3}^{\mu}$ is convenient here as it makes explicit the constraint from lepton current conservation, which implies that for massless leptons $c_{3}^{9}$ does not contribute, while for $\Gamma_{7, i}^{\mu}$ there are only two independent coefficients. Transforming the basis (67) to our operator basis listed in Table 1, the matching coefficients $c_{i}^{9}$ and $c_{i}^{7}$ are given, respectively, as

$$
\begin{aligned}
c_{1}^{9}(u, \mu) & =C_{V}^{1}(u ; \mu), \\
c_{2}^{9}(u, \mu) & =C_{V}^{2}(u ; \mu)+\frac{2}{u} C_{V}^{3}(u ; \mu), \\
c_{3}^{9}(u, \mu) & =-\frac{2}{u m_{b}} C_{V}^{3}(u ; \mu), \\
c_{1}^{7}(u, \mu) & =-2 C_{T}^{1}\left(u ; \mu, \nu=m_{b}\right)+C_{T}^{3}\left(u ; \mu, \nu=m_{b}\right), \\
c_{2}^{7}(u, \mu) & =-\frac{2}{u m_{b}} C_{T}^{3}\left(u ; \mu, \nu=m_{b}\right) .
\end{aligned}
$$

The two-loop matching coefficients $c_{i}^{9}$ for the vector current have become available in the context of inclusive semi-leptonic $B$ decays [9-12]. The results of this paper allow us to compute also the matching coefficients $c_{i}^{7}$ at NNLO. As a consequence the factor in $h^{[0]}$ related to the QCD current matching is now complete at NNLO, while the other factor related to $C_{i}^{\text {incl }}\left(q^{2}, \mu\right)$ is known at the next-to-next-to-leading logarithmic (NNLL) order, since the three-loop $\mathcal{O}\left(\alpha_{s}^{2}\right)$ matrix elements of the current-current operators (giving rise to charm-loop diagrams) are not available.

In Figure [5 we show these matching coefficients as a function of $u$ in the one- (dashed) and two-loop (solid) approximation, evaluated at $\mu=m_{b}=4.8 \mathrm{GeV}$ (blue/dark grey curves) and at $\mu=1.5 \mathrm{GeV}$ (orange/light grey curves), respectively. The difference between these two different choices of the IR factorization scale $\mu$ is compensated by the corresponding scale dependence of the convolution $J \otimes S$ such that the differential rate (63) is $\mu$-independent. Note that, while we show the entire range of $u$, Eq. (66) implies that the relevant values of $u$ for $b \rightarrow s \ell^{+} \ell^{-}$in the $q^{2}$ region of interest are above $u \approx 0.75$. In the lower right panel of Figure 5 , we also show the ratio $c_{1}^{7} / c_{1}^{9}$, which equals the quantity $R_{\perp}$ defined earlier in (58) at $\nu=m_{b}$, and plays an important role for the forward-backward asymmetry as discussed below. Note that $R_{\perp}$ is $\mu$-independent, except for the truncation of the perturbative series. In evaluating this ratio to a given order in $\alpha_{s}$, we expand the denominator and truncate the expanded expression.

Comparing the dashed (one-loop approximation) and solid (two-loop approximation) curves of the same colour in Figure 5, we observe that the two-loop corrections are generally moderate in the large $u$ (low $q^{2}$ ) region, whereas the large correction in the region of small $u$ is due to the fact that increasing powers of large logarithms take over in this region. However, the correction is amplified in the ratio $R_{\perp}$, where the two-loop correction exceeds the one-loop term. This leads to a considerable residual $\mu$-dependence (difference of blue/dark grey and orange/light grey curves) as can also be seen in Figure2. Since the infrared physics drops out from the ratio $c_{1}^{7} / c_{1}^{9}$ the natural scale is of order of the hard scale $m_{b}$. 

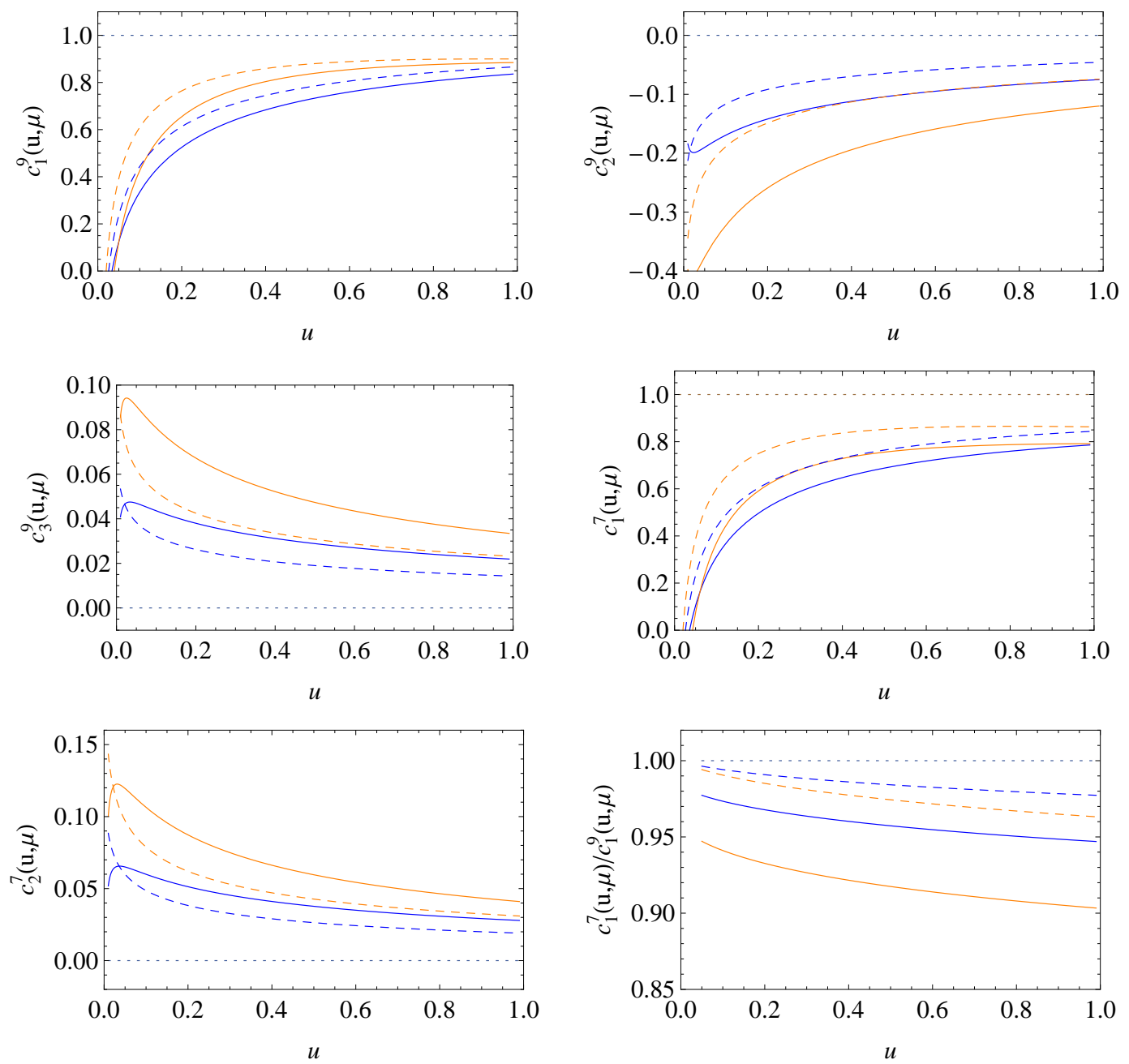

Figure 5: The matching coefficients $c_{i}^{9}(u, \mu)$ and $c_{i}^{7}(u, \mu)$ as a function of $u$ (related to the dilepton invariant mass $q^{2}=(1-u) m_{b}^{2}$ ) in the one-loop (dashed) and two-loop (solid) approximation. The blue/dark grey curves refer to $\mu=m_{b}=4.8 \mathrm{GeV}$, and the orange/light grey ones to $\mu=1.5 \mathrm{GeV}$.

The differential decay rate $(63)$ can be written as

$$
\begin{aligned}
\frac{d^{3} \Gamma}{d q^{2} d p_{X}^{+} d \cos \theta}= & \frac{3}{8}\left[\left(1+\cos ^{2} \theta\right) H_{T}\left(q^{2}, p_{X}^{+}\right)+2\left(1-\cos ^{2} \theta\right) H_{L}\left(q^{2}, p_{X}^{+}\right)\right. \\
& \left.+2 \cos \theta H_{A}\left(q^{2}, p_{X}^{+}\right)\right]
\end{aligned}
$$

where for $\bar{B}$ decay, $\theta$ denotes the angle between the positively charged lepton and the $\bar{B}$ meson in the centre-of-mass frame of the $\ell^{+} \ell^{-}$pair. For fixed $p_{X}^{+}$, the forward-backward asymmetry in $\theta$ therefore vanishes for a particular $q_{0}^{2}$ at which $H_{A}\left(q_{0}^{2}, p_{X}^{+}\right)=0$. Integrating over the invariant mass of the hadronic final state up to the cut $m_{X}^{\text {cut }}$, the asymmetry 
zero occurs at

$$
\begin{aligned}
0 & =\int_{0}^{p_{X}^{+\mathrm{cut}}} d p_{X}^{+} H_{A}\left(q_{0}^{2}, p_{X}^{+}\right) \\
& =\text {const } \times \int_{0}^{p_{X}^{+\mathrm{cut}}} d p_{X}^{+} h_{A}^{[0]}\left(q_{0}^{2}, p_{X}^{+}\right) \frac{\left(q_{0+}-q_{0-}\right)^{2}}{q_{0+}} q_{0}^{2} \int d \omega p^{-} J\left(p^{-} \omega\right) S\left(p_{X}^{+}-\omega\right),
\end{aligned}
$$

where [37] $q_{+}=m_{B}-p_{X}^{+}, q_{-}=q^{2} / q_{+}$,

$$
p_{X}^{+ \text {cut }}=\frac{1}{2 m_{B}}\left[m_{B}^{2}+\left(m_{X}^{\text {cut }}\right)^{2}-q^{2}-\sqrt{\left(m_{B}^{2}+\left(m_{X}^{\text {cut }}\right)^{2}-q^{2}\right)^{2}-4 m_{B}^{2}\left(m_{X}^{\text {cut }}\right)^{2}}\right],
$$

and

$$
h_{A}^{[0]}\left(q^{2}, p_{X}^{+}\right)=2 \mathcal{C}_{10} c_{1}^{9}(u) \operatorname{Re}\left[C_{9}^{\text {incl }}\left(q^{2}\right) c_{1}^{9}(u)+\frac{2 m_{b}}{q_{-}} C_{7}^{\text {incl }}\left(q^{2}\right) c_{1}^{7}(u)\right] .
$$

We now observe that $h_{A}^{[0]}\left(q_{0}^{2}, p_{X}^{+}\right)$depends on $p_{X}^{+}$only through the definition of $u$ in (66) and the kinematic factor $2 m_{b} / q_{-}$. For typical $m_{X}^{\text {cut }}$ of $2 \mathrm{GeV}$ this dependence is very weak, since then $p_{X}^{+} \sim 1 \mathrm{GeV} \ll m_{B}$. Thus, $p_{X}^{+}$appears only as a small correction to $m_{B}-p_{X}^{+}$, and in the definition of $u$ in a term that is additionally suppressed by $q^{2} / m_{B}$ relative to $m_{b}$, see (66). This results in a very small variation of $u$ of about 0.02 over the entire $p_{X}^{+}$integration region. We may therefore pull the slowly varying function $h_{A}^{[0]}\left(q_{0}^{2}, p_{X}^{+}\right)$in front of the $p_{X}^{+}$integration in (70) thereby replacing $p_{X}^{+}$in the argument by an average value which we assume to be $\left\langle p_{X}^{+}\right\rangle=p_{X}^{+ \text {cut }} / 2$. The remaining integral over the jet and soft function is different from zero, thus the forward-backward asymmetry zero is determined by $h_{A}^{[0]}\left(q_{0}^{2},\left\langle p_{X}^{+}\right\rangle\right)=0$. Using (72) this is equivalent to the condition

$$
\frac{q_{0}^{2}}{2 m_{b}\left(m_{B}-\left\langle p_{X}^{+}\right\rangle\right)}=-\frac{\operatorname{Re}\left[C_{7}^{\text {incl }}\left(q_{0}^{2}\right)\right]}{\operatorname{Re}\left[C_{9}^{\text {incl }}\left(q_{0}^{2}\right)\right]} \frac{c_{1}^{7}\left(u_{0}\right)}{c_{1}^{9}\left(u_{0}\right)}
$$

with $u_{0} \equiv 1-q_{0}^{2} /\left(m_{b}\left(m_{B}-\left\langle p_{X}^{+}\right\rangle\right)\right)$. This result leads to the important conclusion that the QCD dynamics that determines the location of the asymmetry zero is to a very good approximation independent of the long-distance physics below the scale $m_{b}$ contained in the jet function and the non-perturbative shape function. It also depends only very weakly on the value of the invariant mass cut through the dependence of $\left\langle p_{X}^{+}\right\rangle$on $m_{X}^{\text {cut }}$. The bulk dependence of $q_{0}^{2}$ on the invariant mass cut $m_{X}^{\text {cut }}$ enters through the kinematical factor $m_{B}-\left\langle p_{X}^{+}\right\rangle$on the left-hand side of (73).

We are now in the position to quantify the impact of the two-loop calculation of $R_{\perp}\left(u_{0}, \nu=m_{b}\right)=c_{1}^{7}\left(u_{0}\right) / c_{1}^{9}\left(u_{0}\right)$ on $q_{0}^{2}$. In [37] the asymmetry zero has been determined by keeping the full NNLL expression for $\operatorname{Re}\left[C_{7}^{\text {incl }}\left(q^{2}\right)\right] / \operatorname{Re}\left[C_{9}^{\text {incl }}\left(q^{2}\right)\right]$ but setting $R_{\perp}=1$. In this approximation, and excluding $1 / m_{b}$-suppressed shape function effects for the moment, the zero is found to be

$$
\left.q_{0}^{2}\right|_{R_{\perp}=1}=(3.62 \ldots 3.69) \mathrm{GeV}^{2} \quad \text { for } \quad m_{X}^{\text {cut }}=(2.0 \ldots 1.8) \mathrm{GeV}
$$




\begin{tabular}{|l|l|}
\hline$\alpha_{s}\left(M_{Z}\right)=0.1180$ & $\lambda_{2} \simeq \frac{1}{4}\left(m_{B^{*}}^{2}-m_{B}^{2}\right) \simeq 0.12 \mathrm{GeV}^{2}$ \\
$\sin ^{2} \theta_{W}=0.23122$ & $m_{t}^{\text {pole }}=171.4 \mathrm{GeV}$ \\
$M_{W}=80.426 \mathrm{GeV}$ & $m_{c}^{\text {pole }}=(1.5 \pm 0.1) \mathrm{GeV}$ \\
$M_{Z}=91.1876 \mathrm{GeV}$ & $m_{b}^{\text {PS }}(2 \mathrm{GeV})=(4.6 \pm 0.1) \mathrm{GeV}$ \\
\hline
\end{tabular}

Table 2: Numerical inputs that we use in the phenomenological analysis of the forward-backward asymmetry zero.

As indicated the lowest value corresponds to $m_{X}^{\text {cut }}=2.0 \mathrm{GeV}$ and the highest one to $m_{X}^{\text {cut }}=1.8 \mathrm{GeV}$. Our value is somewhat larger than what can be extracted from Figure 4 of [37], because we expand the factor $\bar{m}_{b}(\mu) / m_{b}^{\text {pole }}$ that accompanies $C_{7}$ in $\alpha_{s}$. Moreover, the variation of the zero when changing $m_{X}^{\text {cut }}$ from $1.8 \mathrm{GeV}$ to $2.0 \mathrm{GeV}$ is about twice as large compared to what can be read off from Fig. 4 of [37], which is likely due to our approximation of pulling the slowly varying function $h_{A}^{[0]}\left(q_{0}^{2}, p_{X}^{+}\right)$out of the integral in (70). However, our approximation is still justified since even the increased sensitivity of the zero on $m_{X}^{\text {cut }}$ is only $\pm 0.03 \mathrm{GeV}^{2}$ and hence below $1 \%$. Taking into account $R_{\perp}$ at the NLO, we find for the position of the zero

$$
\left.q_{0}^{2}\right|_{R_{\perp} \mathrm{NLO}}=(3.55 \ldots 3.61) \mathrm{GeV}^{2} \quad \text { for } \quad m_{X}^{\text {cut }}=(2.0 \ldots 1.8) \mathrm{GeV} .
$$

The impact of the NLO correction to $R_{\perp}$ is to shift the zero by $-2.2 \%$. As we already stated before, and as can also be seen from Figures 2 and 5 , the size of the NNLO correction to $R_{\perp}$ is significant. It amounts to a shift of the NLO zero in (75) by another $-3 \%$ and hence is larger than the NLO shift. The total shift induced by $R_{\perp}$ through NNLO therefore amounts to $-5 \%$.

Before proceeding to our final result we briefly comment on the rôle of power corrections. The authors of [37] performed a thorough study of $1 / m_{b}$-suppressed shape function effects which result in a shift of the zero of $-0.05 \mathrm{GeV}^{2}$ to $-0.1 \mathrm{GeV}^{2}$. This shift is more strongly dependent on the invariant mass cut and the theoretical error increases when $m_{X}^{\text {cut }}$ is chosen smaller. In the following we take the larger value as an estimate for the shift and also for the associated uncertainty. However, the study of power corrections in [37] does not cover all such corrections and applies a rather crude treatment to those arising from soft gluon attachments to the charm-loop diagrams by absorbing the $1 / \mathrm{m}_{c}^{2}$ non-perturbative power corrections into the $C_{i}^{\text {incl }}$, which is justified only in the absence of invariant mass cuts. In the semi-inclusive region, the matrix element of (29) in 33. cannot, due to the presence of a soft gluon, be expressed in terms of a short-distance coefficient times a local matrix element, since the soft gluon attached to the charm loop affects the invariant mass of an energetic hadronic final state by a relevant amount $\sqrt{m_{b} \Lambda_{\mathrm{QCD}}}$, which must be accounted for by a subleading shape function. By treating this correction 
as in the inclusive case, the authors of [37] implicitly assumed that this shape function somehow factorizes into the local heavy-quark effective theory matrix element $\lambda_{2}$ and the leading-power shape function. It is not clear to us how this simplification can be justified and it is likely not even parametrically correct. Nevertheless, in the absence of better information we follow the treatment of [37] and include the $1 / m_{c}^{2}$ power corrections into the $C_{i}^{\text {incl }}$. This results in a shift of the asymmetry zero by $+0.07 \mathrm{GeV}^{2}$, which is included in (74), (75) , and below in (76). To be conservative we assign another $0.1 \mathrm{GeV}^{2}$ uncertainty to this estimate and add it in quadrature with the other power correction uncertainty.

We are now in the position to present our final NNLO result based on the numerical input parameters and their respective intervals as specified in Table 2, We then find

$$
\begin{aligned}
q_{0}^{2} & =\left[(3.34 \ldots 3.40)_{-0.13 \mu}^{+0.04} \pm 0.08_{m_{b}}^{+0.05}{ }_{-04}^{+0.05} m_{c} \pm 0.14_{\mathrm{SF}} \pm 0.14_{\left\langle p_{X}^{+}\right\rangle}\right] \mathrm{GeV}^{2} \\
& =\left[(3.34 \ldots 3.40)_{-0.25}^{+0.22}\right] \mathrm{GeV}^{2} \quad \text { for } \quad m_{X}^{\text {cut }}=(2.0 \ldots 1.8) \mathrm{GeV}
\end{aligned}
$$

The error estimate is computed as follows: The range of scale variation is taken to be $2.3 \mathrm{GeV}<\mu<9.2 \mathrm{GeV}$, and we vary the scale in the $C_{i}^{\text {incl }}$ and in $R_{\perp}$ independently to account conservatively for the absence of the $\mathcal{O}\left(\alpha_{s}^{2}\right)$ correction to the $C_{i}^{\text {incl }}$. The input quark mass is the bottom mass in the potential-subtracted (PS) scheme [38, see Table 2. The pole mass and $\overline{\mathrm{MS}}$ mass used in intermediate expressions are computed using the one-loop conversion factors resulting in $m_{b}^{\text {pole }}=4.78 \mathrm{GeV}$ and $\bar{m}\left(m_{b}^{\mathrm{PS}}\right)=4.36 \mathrm{GeV}$, respectively, when $m_{b}^{\mathrm{PS}}(2 \mathrm{GeV})=4.6 \mathrm{GeV}$. The dependence on the charm quark mass enters through the matrix elements of the current-current operators. The error labelled "SF" is connected with the subleading shape function effects as discussed above. Finally we have added an uncertainty estimate for the approximation made by pulling out the slowly varying function $h_{A}^{[0]}\left(q_{0}^{2}, p_{X}^{+}\right)$out of the $p_{X}^{+}$integral in (70). We estimate this error rather generously by varying $\left\langle p_{X}^{+}\right\rangle$from $p_{X}^{\text {cut }} / 4$ to $3 p_{X}^{\text {cut }} / 4$. The total error is obtained by adding all these uncertainties in quadrature.

We note that the value of the asymmetry zero in semi-inclusive $b \rightarrow s \ell^{+} \ell^{-}$decay is significantly smaller than for the exclusive case [29], where spectator scattering is

responsible for a positive shift as is the fact that in this case $\left\langle p_{X}^{+}\right\rangle=0$ in (73). On the other hand the semi-inclusive zero is in the same region as in the inclusive case [39], where virtual effects together with hard gluon bremsstrahlung encoded in functions $\omega_{710}$ and $\omega_{910}$ [40] also induce a negative shift on the zero.

\section{Conclusion}

In this paper we completed the two-loop matching calculation for heavy-to-light currents from QCD onto SCET for the complete set of Dirac structures. These matching coefficients enter several phenomenological applications, of which we have discussed their effects on heavy-to-light form factor ratios, exclusive radiative and semi-leptonic decays, as well as the inclusive decay $\bar{B} \rightarrow X_{s} \ell^{+} \ell^{-}$in the shape-function region. The two-loop 
corrections are generally relatively small, in the few percent range. However, one ratio, $R_{\perp}=c_{1}^{7}(u, \mu) / c_{1}^{9}(u, \mu)$, which is also the most important for phenomenology, since it enters the comparison of radiative and semi-leptonic decays as well as the forward-backward asymmetry in exclusive and semi-inclusive $b \rightarrow s \ell^{+} \ell^{-}$transition, exhibits a two-loop correction that is larger than the one-loop term. The two-loop term alone shifts the location of the asymmetry zero by about $-0.1 \mathrm{GeV}^{2}$, comparable to the effect of $1 / m_{b}$ suppressed shape functions estimated in [37]. We showed that the location of the asymmetry zero in semi-inclusive $\bar{B} \rightarrow X_{s} \ell^{+} \ell^{-}$with an invariant mass cut is to a very good approximation independent of the long-distance physics below the scale $m_{b}$ contained in the jet function and the non-perturbative shape function, and obtain $q_{0}^{2}=\left(3.34_{-0.25}^{+0.22}\right) \mathrm{GeV}^{2}$ for an invariant mass cut $m_{X}^{\text {cut }}=2.0 \mathrm{GeV}$ as our best estimate for the asymmetry zero. Moreover, we confirm the discrepancy between QCD sum rule and SCET results for the form factor ratio $T_{1} / V$ in the low $q^{2}$ region discussed in [14] and suggest that a dedicated QCD sum rules analysis of deviations from the symmetry limit (rather than the form factors themselves) should be done to clarify the situation.

\section{Acknowledgements}

We would like to thank F. Tackmann and M. Misiak for useful correspondence. This work was supported in part by the DFG Sonderforschungsbereich/Transregio 9 "Computergestützte Theoretische Teilchenphysik" (GB, MB), the Helmholtz alliance "Physics at the Terascale" (TH), and the Alexander-von-Humboldt Stiftung (X.-Q. Li). X.-Q. Li acknowledges hospitality from the Institute of Theoretical Physics, Chinese Academy of Science, where part of this work was performed.

\section{A NLO coefficient functions}

In Section 3.1 we introduced the following set of one-loop coefficient functions,

$$
\begin{aligned}
g_{0}(u)= & -\frac{5}{2}+2 \ln (u), \\
g_{1}(u)= & -\frac{\pi^{2}}{12}+\frac{2}{\bar{u}} \ln (u)-2 \ln ^{2}(u)-2 \operatorname{Li}_{2}(\bar{u}), \\
g_{2}(u)= & \frac{\pi^{2}}{24}+\frac{1}{3} \zeta_{3}+\frac{12(1+\bar{u})+\pi^{2} \bar{u}}{6 \bar{u}} \ln (u)-\frac{2}{\bar{u}}\left(\ln ^{2}(u)+\operatorname{Li}_{2}(\bar{u})\right)+\frac{4}{3} \ln ^{3}(u) \\
& +4 \ln (u) \operatorname{Li}_{2}(\bar{u})-2 \operatorname{Li}_{3}(\bar{u})+4 \mathrm{~S}_{1,2}(\bar{u}), \\
g_{3}(u)= & -\frac{\pi^{4}}{160}-\frac{1}{6} \zeta_{3}+\frac{48(1+\bar{u})+2 \pi^{2}-8 \bar{u} \zeta_{3}}{12 \bar{u}} \ln (u)-\frac{2}{3} \ln ^{4}(u)-4 \ln ^{2}(u) \operatorname{Li}_{2}(\bar{u}) \\
& -\frac{12(1+\bar{u})+\pi^{2} \bar{u}}{6 \bar{u}}\left(\ln ^{2}(u)+\operatorname{Li}_{2}(\bar{u})\right)-8 \ln (u) \mathrm{S}_{1,2}(\bar{u})+4 \ln (u) \operatorname{Li}_{3}(\bar{u})-2 \operatorname{Li}_{4}(\bar{u}) \\
& +\frac{2}{\bar{u}}\left(\frac{2}{3} \ln ^{3}(u)+2 \ln (u) \operatorname{Li}_{2}(\bar{u})-\operatorname{Li}_{3}(\bar{u})+2 \mathrm{~S}_{1,2}(\bar{u})\right)-8 \mathrm{~S}_{1,3}(\bar{u})+4 \mathrm{~S}_{2,2}(\bar{u}),
\end{aligned}
$$




$$
\begin{aligned}
g_{4}(u)= & g_{1}(u)-6-\frac{4 u}{\bar{u}} \ln (u), \\
g_{5}(u)= & g_{2}(u)-10-\frac{\pi^{2}}{3}-\frac{2 u}{\bar{u}}\left(3 \ln (u)-2 \ln ^{2}(u)-2 \operatorname{Li}_{2}(\bar{u})\right), \\
g_{6}(u)= & g_{3}(u)-18-\frac{\pi^{2}}{2}+\frac{4}{3} \zeta_{3}-\frac{2 u}{\bar{u}}\left(\frac{30+\pi^{2}}{6} \ln (u)-3 \ln ^{2}(u)-3 \operatorname{Li}_{2}(\bar{u})+\frac{4}{3} \ln ^{3}(u)\right. \\
& \left.+4 \ln (u) \operatorname{Li}_{2}(\bar{u})-2 \operatorname{Li}_{3}(\bar{u})+4 \mathrm{~S}_{1,2}(\bar{u})\right) \\
g_{7}(u)= & -\frac{2}{\bar{u}}-\frac{2 u}{\bar{u}^{2}} \ln (u), \\
g_{8}(u)= & -\frac{6}{\bar{u}}-\frac{2 u}{\bar{u}^{2}}\left(2 \ln (u)-\ln (u)-\operatorname{Li}_{2}(\bar{u})\right), \\
g_{9}(u)= & \frac{2 u}{\bar{u}} \ln (u), \\
g_{10}(u)= & \frac{u(1+4 \bar{u})}{\bar{u}^{2}} \ln (u)+\frac{u}{\bar{u}}\left(1-2 \ln ^{2}(u)-2 \operatorname{Li}_{2}(\bar{u})\right), \\
g_{13}(u)= & g_{8}(u)+6+\frac{2 u}{\bar{u}} \ln (u) . \\
g_{11}(u)= & \frac{6 u(2+7 \bar{u})+\pi^{2} u \bar{u}}{6 \bar{u}^{2}} \ln (u)-\frac{u(1+4 \bar{u})}{\bar{u}^{2}}\left(\ln ^{2}(u)+\operatorname{Li}_{2}(\bar{u})\right) \\
& +\frac{u}{\bar{u}}\left(3+\frac{4}{3} \ln { }^{3}(u)+4 \ln (u) \operatorname{Li}_{2}(\bar{u})-2 \operatorname{Li}_{3}(\bar{u})+4 \mathrm{~S}_{1,2}(\bar{u})\right), \\
g_{7}(u)+2 & \\
&
\end{aligned}
$$

\section{B NNLO coefficient functions}

The finite parts of the two-loop form factors involve the following coefficient functions,

$$
\begin{aligned}
h_{1}(u) & =-\frac{2\left(7-2 \bar{u}+3 \bar{u}^{2}\right)}{u^{2}} \operatorname{Li}_{4}(\bar{u})-\frac{4\left(11+2 \bar{u}+3 \bar{u}^{2}\right)}{u^{2}} \mathrm{~S}_{2,2}(\bar{u})+8 \mathrm{~S}_{1,3}(\bar{u})-8 \ln (u) \operatorname{Li}_{3}(\bar{u}) \\
& +\frac{2\left(3+\bar{u}^{2}\right)}{u^{2}} \operatorname{Li}_{2}(\bar{u})^{2}+16 \ln (u) \mathrm{S}_{1,2}(\bar{u})+\frac{16}{3} \ln ^{4}(u)+16 \ln ^{2}(u) \operatorname{Li}_{2}(\bar{u}) \\
& -\frac{6+47 \bar{u}-5 \bar{u}^{2}}{3 u \bar{u}} \operatorname{Li}_{3}(\bar{u})-\frac{2(42-29 \bar{u})}{9 \bar{u}} \ln ^{3}(u)+\frac{2\left(6-115 \bar{u}+13 \bar{u}^{2}\right)}{3 u \bar{u}} \mathrm{~S}_{1,2}(\bar{u}) \\
& -\frac{2\left(12+\bar{u}+11 \bar{u}^{2}\right)}{3 u \bar{u}} \ln (u) \operatorname{Li}_{2}(\bar{u})+\frac{36-87 \bar{u}-250 \bar{u}^{2}+18 \pi^{2} \bar{u}^{2}}{9 \bar{u}^{2}} \ln ^{2}(u) \\
& -\left(\frac{33+109 \bar{u}-322 \bar{u}^{2}}{9 u \bar{u}}-\frac{\left(7-2 \bar{u}+3 \bar{u}^{2}\right) \pi^{2}}{u^{2}}\right) \operatorname{Li}_{2}(\bar{u})+\frac{(2815+353 \bar{u}) \pi^{2}}{432 u}
\end{aligned}
$$




$$
\begin{aligned}
& +\left(\frac{1173+241 \bar{u}}{27 \bar{u}}-\frac{\left(9+46 \bar{u}+17 \bar{u}^{2}\right) \pi^{2}}{9 u \bar{u}}-\frac{56}{3} \zeta_{3}\right) \ln (u) \\
& -\frac{\left(509+278 \bar{u}+77 \bar{u}^{2}\right) \pi^{4}}{720 u^{2}}+\frac{76}{9} \zeta_{3}+\frac{30331}{1296}-2 h_{2}(u), \\
& h_{2}(u)=\frac{2(1+\bar{u})^{2}}{3 u \bar{u}}\left(12 \mathcal{H}_{1}(\bar{u})+\pi^{2} \ln (2-u)\right)+\frac{1}{3}\left(24 \mathcal{H}_{2}(\bar{u})-2 \pi^{2} \operatorname{Li}_{2}(-\bar{u})\right) \\
& -\frac{8}{u^{2}} \mathrm{~S}_{2,2}(\bar{u})-\frac{2}{u^{2}} \operatorname{Li}_{4}(\bar{u})-8 \ln (u) \operatorname{Li}_{3}(\bar{u})-\frac{(u-\bar{u})(3-2 \bar{u})}{u^{2}} \operatorname{Li}_{2}(\bar{u})^{2} \\
& -\frac{40-56 \bar{u}+7 \bar{u}^{2}}{3 u^{2}} \operatorname{Li}_{3}(\bar{u})+\frac{14-40 \bar{u}+17 \bar{u}^{2}}{3 u^{2}} \mathrm{~S}_{1,2}(\bar{u})+\frac{29-35 \bar{u}}{3 u} \ln (u) \operatorname{Li}_{2}(\bar{u}) \\
& +\frac{44}{9} \ln ^{3}(u)-\left(\frac{66+122 \bar{u}-89 \bar{u}^{2}}{9 u \bar{u}}-\frac{\left(7-8 \bar{u}+4 \bar{u}^{2}\right) \pi^{2}}{3 u^{2}}\right) \operatorname{Li}_{2}(\bar{u}) \\
& -\frac{78+223 \bar{u}-12 \pi^{2} \bar{u}}{18 \bar{u}} \ln ^{2}(u)+\frac{\left(13-62 \bar{u}+31 \bar{u}^{2}\right) \pi^{4}}{120 u^{2}} \\
& +\left(\frac{2(354+121 \bar{u})}{27 \bar{u}}-\frac{\left(24-71 \bar{u}+65 \bar{u}^{2}\right) \pi^{2}}{18 u \bar{u}}-14 \zeta_{3}\right) \ln (u) \\
& +\frac{3(2-\bar{u})^{2}}{u^{2}}\left(\operatorname{Li}_{3}(-u)-\ln (u) \operatorname{Li}_{2}(-u)-\frac{\ln ^{2}(u)+\pi^{2}}{2} \ln (1+u)\right)+\frac{5405}{1296} \\
& +\frac{(877+239 \bar{u}) \pi^{2}}{216 u}+\frac{469-73 \bar{u}}{18 u} \zeta_{3}-\frac{2(3+\bar{u}) \pi^{2}}{u} \ln (2), \\
& h_{3}(u)=\frac{8}{3} \operatorname{Li}_{3}(\bar{u})+\frac{8(1+\bar{u})\left(3+11 \bar{u}-11 \bar{u}^{2}+5 \bar{u}^{3}\right)}{9 u^{3} \bar{u}} \operatorname{Li}_{2}(\bar{u}) \\
& -\frac{2\left(96+208 \bar{u}-224 \bar{u}^{2}+112 \bar{u}^{3}+3 \bar{u} u^{2} \pi^{2}\right)}{27 u^{2} \bar{u}} \ln (u)+\frac{3773-4954 \bar{u}+2333 \bar{u}^{2}}{81 u^{2}} \\
& -\frac{\left(265-315 \bar{u}+219 \bar{u}^{2}-41 \bar{u}^{3}\right) \pi^{2}}{54 u^{3}}-\frac{28}{9} \zeta_{3}, \\
& h_{4}(u)=h_{1}(u)+2 h_{2}(u)+\frac{12(1+\bar{u})^{2}}{u^{3}}\left(8 \mathrm{~S}_{2,2}(\bar{u})+2 \mathrm{Li}_{4}(\bar{u})-\operatorname{Li}_{2}(\bar{u})^{2}+\frac{3 \pi^{4}}{20}\right) \\
& +\frac{4(1+\bar{u})\left(1+10 \bar{u}+\bar{u}^{2}\right)}{u^{2} \bar{u}} \operatorname{Li}_{3}(\bar{u})+\frac{56 u}{3 \bar{u}} \ln ^{3}(u)-\frac{8\left(1-31 \bar{u}-13 \bar{u}^{2}-5 \bar{u}^{3}\right)}{u^{2} \bar{u}} \mathrm{~S}_{1,2}(\bar{u}) \\
& +\frac{8\left(2+\bar{u}+10 \bar{u}^{2}-\bar{u}^{3}\right)}{u^{2} \bar{u}} \ln (u) \operatorname{Li}_{2}(\bar{u})-\frac{4\left(3-4 \bar{u}-46 \bar{u}^{2}+11 \bar{u}^{3}\right)}{3 u \bar{u}^{2}} \ln ^{2}(u) \\
& +\left(\frac{4\left(4+3 \bar{u}-72 \bar{u}^{2}-7 \bar{u}^{3}\right)}{3 u^{2} \bar{u}}-\frac{12(1+\bar{u})^{2} \pi^{2}}{u^{3}}\right) \operatorname{Li}_{2}(\bar{u})-\frac{\left(359+362 \bar{u}+143 \bar{u}^{2}\right) \pi^{2}}{18 u^{2}} \\
& -\left(\frac{611-251 \bar{u}}{9 \bar{u}}-\frac{2\left(3+32 \bar{u}+35 \bar{u}^{2}+2 \bar{u}^{3}\right) \pi^{2}}{3 u^{2} \bar{u}}\right) \ln (u)-\frac{4}{3} \zeta_{3}-\frac{3050}{27}-2 h_{5}(u),
\end{aligned}
$$




$$
\begin{aligned}
& h_{5}(u)=h_{2}(u)-\frac{4(1+\bar{u})}{3 \bar{u}}\left(12 \mathcal{H}_{1}(\bar{u})+\pi^{2} \ln (2-u)\right)+\frac{4\left(12-21 \bar{u}+18 \bar{u}^{2}-8 \bar{u}^{3}\right)}{3 u^{3}} \operatorname{Li}_{3}(\bar{u}) \\
& +\frac{2\left(1+3 \bar{u}^{2}\right)}{u^{3}}\left(8 \mathrm{~S}_{2,2}(\bar{u})+2 \operatorname{Li}_{4}(\bar{u})-\operatorname{Li}_{2}(\bar{u})^{2}+\frac{3 \pi^{4}}{20}\right)+\frac{16\left(1+\bar{u}+\bar{u}^{2}\right)}{3 u^{2}} \ln (u) \operatorname{Li}_{2}(\bar{u}) \\
& +\frac{4\left(14+15 \bar{u}-24 \bar{u}^{2}-6 \bar{u}^{3}\right)}{3 u^{3}} \mathrm{~S}_{1,2}(\bar{u})-\left(\frac{472 u}{9 \bar{u}}-\frac{8\left(1-2 \bar{u}+4 \bar{u}^{2}\right) \pi^{2}}{3 u^{2} \bar{u}}\right) \ln (u) \\
& +\left(\frac{4\left(11-21 \bar{u}+4 \bar{u}^{2}-5 \bar{u}^{3}\right)}{3 u^{2} \bar{u}}-\frac{2\left(1+3 \bar{u}^{2}\right) \pi^{2}}{u^{3}}\right) \operatorname{Li}_{2}(\bar{u}) \\
& -\frac{4(2-\bar{u})\left(5-8 \bar{u}+2 \bar{u}^{2}\right)}{3 u^{3}}\left(\operatorname{Li}_{3}(-u)-\ln (u) \operatorname{Li}_{2}(-u)-\frac{\ln ^{2}(u)+\pi^{2}}{2} \ln (1+u)\right) \\
& +\frac{2\left(13-18 \bar{u}+16 \bar{u}^{2}\right)}{3 u \bar{u}} \ln ^{2}(u)-\frac{2\left(13+4 \bar{u}+16 \bar{u}^{2}\right) \pi^{2}}{9 u^{2}}-\frac{16}{u} \zeta_{3}-\frac{5219}{54}, \\
& h_{6}(u)=-\frac{8 u}{3 \bar{u}} \ln (u)+\frac{10}{3} \zeta_{3}+\frac{11 \pi^{2}}{18}-\frac{1381}{324} \\
& h_{7}(u)=h_{3}(u)-\frac{16(1+\bar{u})^{3}}{3 u^{2} \bar{u}} \operatorname{Li}_{2}(\bar{u})+\frac{128\left(1+\bar{u}+\bar{u}^{2}\right)}{9 u \bar{u}} \ln (u)+\frac{32(1+\bar{u}) \pi^{2}}{9 u^{2}} \\
& -\frac{2(251+325 \bar{u})}{27 u} \\
& h_{8}(u)=-\frac{2\left(3+20 \bar{u}+13 \bar{u}^{2}\right)}{u^{3}}\left(8 \mathrm{~S}_{2,2}(\bar{u})+2 \operatorname{Li}_{4}(\bar{u})-\operatorname{Li}_{2}(\bar{u})^{2}+\frac{3 \pi^{4}}{20}\right)-\frac{28 u}{3 \bar{u}} \ln ^{3}(u) \\
& -\frac{2\left(1+17 \bar{u}+51 \bar{u}^{2}+3 \bar{u}^{3}\right)}{u^{2} \bar{u}} \operatorname{Li}_{3}(\bar{u})+\frac{4\left(1-43 \bar{u}-93 \bar{u}^{2}-9 \bar{u}^{3}\right)}{u^{2} \bar{u}} \mathrm{~S}_{1,2}(\bar{u}) \\
& -\frac{8\left(1+2 \bar{u}+15 \bar{u}^{2}\right)}{u^{2} \bar{u}} \ln (u) \operatorname{Li}_{2}(\bar{u})+\frac{9+13 \bar{u}-209 \bar{u}^{2}-29 \bar{u}^{3}}{3 u \bar{u}^{2}} \ln ^{2}(u) \\
& +\left(\frac{3-14 \bar{u}-84 \bar{u}^{2}+402 \bar{u}^{3}+125 \bar{u}^{4}}{3 u^{2} \bar{u}^{2}}+\frac{2\left(3+20 \bar{u}+13 \bar{u}^{2}\right) \pi^{2}}{u^{3}}\right) \operatorname{Li}_{2}(\bar{u}) \\
& -\left(\frac{81-539 \bar{u}+242 \bar{u}^{2}}{18 \bar{u}^{2}}+\frac{\left(1+17 \bar{u}+51 \bar{u}^{2}+3 \bar{u}^{3}\right) \pi^{2}}{u^{2} \bar{u}}\right) \ln (u) \\
& +\frac{2\left(14+77 \bar{u}+17 \bar{u}^{2}\right) \pi^{2}}{3 u^{2}}-\frac{11-3 \bar{u}}{2 \bar{u}}-2 h_{9}(u) \\
& h_{9}(u)=\frac{1}{2} h_{2}(u)-\frac{1}{2} h_{5}(u)-\frac{2 \bar{u}(1+3 \bar{u})}{u^{3}}\left(8 \mathrm{~S}_{2,2}(\bar{u})+2 \mathrm{Li}_{4}(\bar{u})-\operatorname{Li}_{2}(\bar{u})^{2}+\frac{3 \pi^{4}}{20}\right) \\
& -\frac{2\left(3+21 \bar{u}-24 \bar{u}^{2}+2 \bar{u}^{3}\right)}{3 u^{3}} \operatorname{Li}_{3}(\bar{u})-\frac{4\left(1+7 \bar{u}+4 \bar{u}^{2}\right)}{3 u^{2}} \ln (u) \operatorname{Li}_{2}(\bar{u}) \\
& -\frac{2\left(1+69 \bar{u}-48 \bar{u}^{2}-24 \bar{u}^{3}\right)}{3 u^{3}} \mathrm{~S}_{1,2}(\bar{u})+\left(4+\frac{2\left(1-11 \bar{u}-2 \bar{u}^{2}\right) \pi^{2}}{3 u^{2}}\right) \ln (u)
\end{aligned}
$$




$$
\begin{gathered}
-\left(\frac{2\left(9-13 \bar{u}-18 \bar{u}^{2}\right)}{3 u^{2}}-\frac{2 \bar{u}(1+3 \bar{u}) \pi^{2}}{u^{3}}\right) \operatorname{Li}_{2}(\bar{u})-\frac{7+15 \bar{u}}{3 u} \ln ^{2}(u) \\
-\frac{2\left(1-3 \bar{u}+6 \bar{u}^{2}-2 \bar{u}^{3}\right)}{3 u^{3}}\left(\operatorname{Li}_{3}(-u)-\ln (u) \operatorname{Li}_{2}(-u)-\frac{\ln ^{2}(u)+\pi^{2}}{2} \ln (1+u)\right) \\
+\frac{\left(11+17 \bar{u}+38 \bar{u}^{2}\right) \pi^{2}}{9 u^{2}}+\frac{4(3-\bar{u})}{u} \zeta_{3}-4 \pi^{2} \ln (2)-\frac{5435}{108}, \\
h_{10}(u)=\frac{1}{2} h_{3}(u)-\frac{1}{2} h_{7}(u)-\frac{8 \pi^{2}}{9}+\frac{181}{27} .
\end{gathered}
$$

Moreover, for the ratios $R_{X}$ in (58) we need the following auxiliary functions,

$$
\begin{aligned}
& j_{1}(u)=\frac{4(u-2)\left(u^{2}+2 u-2\right)}{3 u^{2} \bar{u}} s_{1}(u)+\frac{16 \bar{u}}{3 u^{3}} s_{2}(u)+\frac{8(2 u-7) \bar{u}}{3 u^{3}} s_{3}(u)-\frac{4 u}{3 \bar{u}} s_{4}(u) \\
& +\frac{2(u+3)\left(u^{2}-1\right)}{u^{3}} s_{5}(u)-\frac{16(2 u-3) \bar{u} \operatorname{Li}_{4}(\bar{u})}{u^{3}}-\frac{4\left(u^{2}-36 u+25\right) \operatorname{Li}_{2}(\bar{u})}{u^{2}} \\
& +\frac{4\left(4 u^{3}-8 u^{2}+5 u+3\right)\left(\operatorname{Li}_{3}(\bar{u})-\zeta(3)\right)}{u^{3}}+\frac{4\left(7 u^{3}+85 u^{2}-111 u+3\right) \operatorname{Li}_{3}(u)}{u^{3}} \\
& +\frac{8 \pi^{2}(6 u-17) \bar{u} \operatorname{Li}_{2}(\bar{u})}{3 u^{3}}+\frac{6 u \operatorname{Li}_{2}(\bar{u})}{\bar{u}}-\frac{4\left(5 u^{3}+63 u^{2}-83 u+3\right) \operatorname{Li}_{2}(u) \ln (u)}{u^{3}} \\
& -\frac{2 \pi^{4}(20 u-33) \bar{u}}{45 u^{3}}-\frac{8\left(u^{2}+51 u-62\right) \zeta(3)}{u^{2}}-\frac{2 \pi^{2}\left(9 u^{2}-73 u+59\right)}{3 u^{2}} \\
& -\frac{32 \pi^{2}(5 u-6) \ln (u)}{3 u^{2}}-\frac{2\left(3 u^{3}+41 u^{2}-55 u+3\right) \ln ^{2}(u) \ln (\bar{u})}{u^{3}} \\
& +\frac{4(2 u-1)^{2} \ln ^{2}(u)}{\bar{u}^{2}}+\frac{(9 u-4) \ln (u)}{\bar{u}}-\frac{2(11 u-25) \ln ^{2}(u)}{u}-1, \\
& j_{2}(u)=-\frac{2(u-2)\left(u^{2}+2 u-2\right)}{3 u^{2} \bar{u}} s_{1}(u)-\frac{8 \bar{u}}{3 u^{3}} s_{2}(u)+\frac{2(4 u-7) \bar{u}}{3 u^{3}} s_{3}(u) \\
& -\frac{(u+3)\left(u^{2}-1\right)}{u^{3}} s_{5}(u)+\frac{2 \pi^{2}(12 u-29) \bar{u} \operatorname{Li}_{2}(\bar{u})}{3 u^{3}}-\frac{4(4 u-15) \bar{u} \operatorname{Li}_{4}(\bar{u})}{u^{3}} \\
& -\frac{2\left(25 u^{2}-99 u+51\right) \operatorname{Li}_{2}(\bar{u})}{3 u^{2}}-\frac{2\left(3 u^{3}+29 u^{2}-37 u+3\right)\left(\operatorname{Li}_{3}(\bar{u})-\zeta(3)\right)}{u^{3}} \\
& -\frac{2\left(11 u^{3}-63 u^{2}+57 u+3\right) \operatorname{Li}_{3}(u)}{u^{3}}+\frac{2\left(8 u^{3}-48 u^{2}+43 u+3\right) \operatorname{Li}_{2}(u) \ln (u)}{u^{3}} \\
& -\frac{22 \mathrm{Li}_{2}(\bar{u})}{3 \bar{u}}-\frac{4 \pi^{4}(5 u-18) \bar{u}}{45 u^{3}}+\frac{2\left(7 u^{2}-83 u+86\right) \zeta(3)}{u^{2}}-\frac{\pi^{2}\left(8 u^{2}-69 u+67\right)}{3 u^{2}} \\
& +\frac{4 \pi^{2}\left(2 u^{2}-17 u+18\right) \ln (u)}{3 u^{2}}+\frac{\left(5 u^{3}-33 u^{2}+29 u+3\right) \ln ^{2}(u) \ln (\bar{u})}{u^{3}}-\frac{13 \ln ^{2}(u)}{3 \bar{u}} \\
& -\frac{4 \pi^{2} \ln (u)}{3 \bar{u}}+\frac{203 \ln (u)}{9 \bar{u}}-\frac{(8 u-51) \ln ^{2}(u)}{3 u}-\frac{257 \ln (u)}{9}+\frac{269}{9},
\end{aligned}
$$




$$
\begin{aligned}
& j_{3}(u)=-\frac{26}{9} g_{9}(u)+\frac{8 u}{3 \bar{u}}\left[\ln ^{2}(u)+\operatorname{Li}_{2}(\bar{u})\right]-\frac{76}{9}, \\
& j_{4}(u)=\frac{32 \pi^{2}(u+2) \bar{u}}{9 u^{2}}+\frac{32 \bar{u}\left(\operatorname{Li}_{3}(\bar{u})-\zeta(3)\right)}{u^{3}}-\frac{8(u-2)\left(u^{2}+2 u-2\right) \mathrm{Li}_{2}(\bar{u})}{3 u^{2} \bar{u}} \\
& -\frac{52 u \ln (u)}{9 \bar{u}}-\frac{104}{3 u}+\frac{80 \ln (u)}{3 u}+\frac{236}{9}, \\
& j_{5}(u)=-\frac{4(u-2)\left(u^{2}-2 u+2\right)}{3 u^{2} \bar{u}} s_{1}(u)+\frac{16 \bar{u}}{3 u^{3}} s_{2}(u)-\frac{8\left(2 u^{2}-12 u+11\right)}{3 u^{3}} s_{3}(u) \\
& +\frac{4}{3 \bar{u}} s_{4}(u)-\frac{2(u+1)\left(u^{2}+2 u+7\right)}{3 u^{3}} s_{5}(u)+\frac{16\left(2 u^{2}-8 u+7\right) \operatorname{Li}_{4}(\bar{u})}{u^{3}} \\
& -\frac{2\left(51 u^{2}-328 u+250\right) \operatorname{Li}_{2}(\bar{u})}{3 u^{2}}-\frac{4\left(u^{3}+48 u^{2}-69 u-7\right)\left(\operatorname{Li}_{3}(\bar{u})-\zeta(3)\right)}{3 u^{3}} \\
& +\frac{4\left(3 u^{3}+315 u^{2}-519 u+7\right) \operatorname{Li}_{3}(u)}{3 u^{3}}-\frac{8 \pi^{2}\left(6 u^{2}-32 u+29\right) \operatorname{Li}_{2}(\bar{u})}{3 u^{3}}-\frac{2 \operatorname{Li}_{2}(\bar{u})}{\bar{u}} \\
& +\frac{4\left(2 u^{3}-237 u^{2}+387 u-7\right) \operatorname{Li}_{2}(u) \ln (u)}{3 u^{3}}+\frac{2 \pi^{4}\left(20 u^{2}-83 u+73\right)}{45 u^{3}} \\
& +\frac{8\left(4 u^{2}-183 u+306\right) \zeta(3)}{3 u^{2}}-\frac{\pi^{2}\left(11 u^{2}-206 u+218\right)}{3 u^{2}}+\frac{8 \pi^{2}\left(u^{2}-72 u+120\right) \ln (u)}{9 u^{2}} \\
& +\frac{2\left(7 u^{3}-159 u^{2}+255 u-7\right) \ln ^{2}(u) \ln (\bar{u})}{3 u^{3}}+\frac{4 \ln ^{2}(u)}{\bar{u}}+\frac{\ln (u)}{\bar{u}}-13 \ln (u) \\
& -\frac{2(27 u-125) \ln ^{2}(u)}{3 u}-8 \pi^{2} \ln (2)+\frac{563}{24}, \\
& j_{6}(u)=\frac{2(u-2)\left(u^{2}-2 u+2\right)}{3 u^{2} \bar{u}} s_{1}(u)-\frac{8 \bar{u}}{3 u^{3}} s_{2}(u)-\frac{2\left(7 u^{2}-17 u+11\right)}{3 u^{3}} s_{3}(u) \\
& +\frac{(u+1)\left(u^{2}+2 u+7\right)}{3 u^{3}} s_{5}(u)+\frac{4\left(7 u^{2}-25 u+19\right) \mathrm{Li}_{4}(\bar{u})}{u^{3}} \\
& -\frac{2\left(35 u^{2}-133 u+73\right) \mathrm{Li}_{2}(\bar{u})}{3 u^{2}}+\frac{2\left(7 u^{3}-99 u^{2}+129 u-7\right)\left(\operatorname{Li}_{3}(\bar{u})-\zeta(3)\right)}{3 u^{3}} \\
& -\frac{2\left(45 u^{3}-273 u^{2}+273 u+7\right) \operatorname{Li}_{3}(u)}{3 u^{3}}-\frac{2 \pi^{2}\left(21 u^{2}-59 u+41\right) \operatorname{Li}_{2}(\bar{u})}{3 u^{3}}+\frac{22 \operatorname{Li}_{2}(\bar{u})}{3 \bar{u}} \\
& +\frac{2\left(28 u^{3}-204 u^{2}+207 u+7\right) \operatorname{Li}_{2}(u) \ln (u)}{3 u^{3}}+\frac{\pi^{4}\left(35 u^{2}-122 u+92\right)}{45 u^{3}} \\
& +\frac{2\left(40 u^{2}-369 u+378\right) \zeta(3)}{3 u^{2}}-\frac{\pi^{2}\left(68 u^{2}-279 u+267\right)}{9 u^{2}}+\frac{4 \pi^{2}\left(8 u^{2}-75 u+78\right) \ln (u)}{9 u^{2}} \\
& +\frac{\left(11 u^{3}-135 u^{2}+141 u+7\right) \ln ^{2}(u) \ln (\bar{u})}{3 u^{3}}+\frac{13 \ln ^{2}(u)}{3 \bar{u}}-\frac{269 \ln (u)}{9 \bar{u}}+\frac{4 \pi^{2} \ln (u)}{3 \bar{u}} \\
& +\frac{215 \ln (u)}{9}-\frac{(40 u-73) \ln ^{2}(u)}{3 u}+4 \pi^{2} \ln (2)-\frac{4421}{216}
\end{aligned}
$$




$$
\begin{aligned}
j_{7}(u) & =\frac{38}{9} g_{9}(u)-\frac{8 u}{3 \bar{u}}\left[\ln ^{2}(u)+\operatorname{Li}_{2}(\bar{u})\right]+\frac{4 \pi^{2}}{9}+\frac{205}{54} \\
j_{8}(u) & =-\frac{8 \pi^{2}\left(u^{2}+8 u-16\right)}{9 u^{2}}+\frac{32 \bar{u}\left(\operatorname{Li}_{3}(\bar{u})-\zeta(3)\right)}{u^{3}}+\frac{8(u-2)\left(u^{2}-10 u+10\right) \operatorname{Li}_{2}(\bar{u})}{3 u^{2} \bar{u}} \\
& +\frac{76 \ln (u)}{9 \bar{u}}-\frac{232}{3 u}-\frac{4(19 u-156) \ln (u)}{9 u}+\frac{1429}{54} \\
j_{9}(u) & =-\frac{5 \pi^{2}(5 u+4)}{6 u}-\frac{16 \bar{u}\left(\operatorname{Li}_{3}(\bar{u})-\zeta(3)\right)}{u^{2}}-\frac{2(u-2) \operatorname{Li}_{2}(\bar{u})}{u}+\frac{u^{2} \ln ^{2}(u)}{\bar{u}^{2}} \\
& -12 \ln (u)-6 \zeta(3)+4 \pi^{2} \ln (2)+\frac{563}{16}, \\
j_{10}(u) & =\frac{4 \pi^{2}(u+1)}{3 u}+\frac{8 \bar{u}\left(\operatorname{Li}_{3}(\bar{u})-\zeta(3)\right)}{u^{2}}+4 \ln (u)+3 \zeta(3)-2 \pi^{2} \ln (2)-\frac{5141}{144}
\end{aligned}
$$

with

$$
\begin{aligned}
& s_{1}(u)=12 \mathcal{H}_{1}(\bar{u})+\pi^{2} \ln (2-u), \\
& s_{2}(u)=12 \mathcal{H}_{2}(\bar{u})-\pi^{2} \operatorname{Li}_{2}(-\bar{u}), \\
& s_{3}(u)=3 \operatorname{Li}_{2}^{2}(\bar{u})-24 \mathrm{~S}_{2,2}(\bar{u})-\frac{17 \pi^{4}}{60}, \\
& s_{4}(u)=6 \operatorname{Li}_{3}(u)-3 \operatorname{Li}_{2}(u) \ln (u)+3 \operatorname{Li}_{3}(\bar{u})-2 \pi^{2} \ln (u)-6 \zeta(3), \\
& s_{5}(u)=-2 \operatorname{Li}_{3}(-u)+2 \operatorname{Li}_{2}(-u) \ln (u)+\ln (u+1) \ln ^{2}(u)+\pi^{2} \ln (u+1) .
\end{aligned}
$$

\section{References}

[1] M. Beneke, G. Buchalla, M. Neubert and C. T. Sachrajda, Phys. Rev. Lett. 83 (1999) 1914 hep-ph/9905312;

M. Beneke, G. Buchalla, M. Neubert and C. T. Sachrajda, Nucl. Phys. B 591 (2000) 313 hep-ph/0006124.

[2] C. W. Bauer, S. Fleming, D. Pirjol and I. W. Stewart, Phys. Rev. D 63 (2001) 114020 [hep-ph/0011336];

C. W. Bauer, D. Pirjol and I. W. Stewart, Phys. Rev. D 65 (2002) 054022 hep-ph/0109045.

[3] M. Beneke, A. P. Chapovsky, M. Diehl and T. Feldmann, Nucl. Phys. B 643 (2002) 431 hep-ph/0206152;

M. Beneke and T. Feldmann, Phys. Lett. B 553 (2003) 267 hep-ph/0211358.

[4] M. Beneke and T. Feldmann, Nucl. Phys. B 685 (2004) 249 hep-ph/0311335. 
[5] C. W. Bauer, D. Pirjol and I. W. Stewart, Phys. Rev. D 67 (2003) 071502 hep-ph/0211069.

[6] B. O. Lange and M. Neubert, Nucl. Phys. B 690 (2004) 249 [Erratum-ibid. B 723 (2005) 201] [hep-ph/0311345].

[7] M. Beneke and T. Feldmann, Nucl. Phys. B 592 (2001) 3 hep-ph/0008255.

[8] M. Beneke, Y. Kiyo and D. s. Yang, Nucl. Phys. B 692 (2004) 232 [hep-ph/0402241].

[9] R. Bonciani and A. Ferroglia, JHEP 0811 (2008) 065, arXiv:0809.4687 [hep-ph].

[10] H. M. Asatrian, C. Greub and B. D. Pecjak, Phys. Rev. D 78 (2008) 114028, arXiv:0810.0987 [hep-ph].

[11] M. Beneke, T. Huber and X. Q. Li, Nucl. Phys. B 811 (2009) 77, arXiv:0810.1230 [hep-ph].

[12] G. Bell, Nucl. Phys. B 812 (2009) 264, arXiv:0810.5695 [hep-ph].

[13] R. J. Hill, T. Becher, S. J. Lee and M. Neubert, JHEP 0407 (2004) 081 hep-ph/0404217.

[14] M. Beneke and D. Yang, Nucl. Phys. B 736 (2006) 34 hep-ph/0508250.

[15] F. V. Tkachov, Phys. Lett. B 100 (1981) 65;

K. G. Chetyrkin and F. V. Tkachov, Nucl. Phys. B 192 (1981) 159.

[16] S. Laporta, Int. J. Mod. Phys. A 15 (2000) 5087 [hep-ph/0102033].

[17] G. Bell, Nucl. Phys. B 795 (2008) 1, arXiv:0705.3127 [hep-ph];

G. Bell, PhD thesis, LMU München, 2006, arXiv:0705.3133 [hep-ph];

G. Bell, Nucl. Phys. B 822 (2009) 172, arXiv:0902.1915 [hep-ph].

[18] T. Huber, JHEP 0903 (2009) 024, arXiv:0901.2133 [hep-ph];

M. Beneke, T. Huber and X. Q. Li, Nucl. Phys. B 832 (2010) 109, arXiv:0911.3655 [hep-ph].

[19] E. Remiddi and J. A. M. Vermaseren, Int. J. Mod. Phys. A 15 (2000) 725 hep-ph/9905237.

[20] J. Blumlein and S. Kurth, Phys. Rev. D 60 (1999) 014018 hep-ph/9810241.

[21] D. V. Nanopoulos and D. A. Ross, Nucl. Phys. B 157 (1979) 273;

R. Tarrach, Nucl. Phys. B 183 (1981) 384;

D. J. Broadhurst and A. G. Grozin, Phys. Rev. D 52 (1995) 4082 [hep-ph/9410240].

[22] T. Becher and M. Neubert, Phys. Lett. B 633 (2006) 739 hep-ph/0512208];

T. Becher and M. Neubert, Phys. Lett. B 637 (2006) 251 [hep-ph/0603140]. 
[23] An electronic file containing all coefficients is attached to the arXiv submission of the present work, and can be obtained from the authors upon request.

[24] A. Ali, B. D. Pecjak and C. Greub, Eur. Phys. J. C 55 (2008) 577, arXiv:0709.4422 [hep-ph].

[25] H. M. Asatrian, A. Hovhannisyan, V. Poghosyan, T. Ewerth, C. Greub and T. Hurth, Nucl. Phys. B 749 (2006) 325 [hep-ph/0605009].

[26] G. Burdman and G. Hiller, Phys. Rev. D 63 (2001) 113008 [hep-ph/0011266].

[27] P. Ball and R. Zwicky, Phys. Rev. D 71 (2005) 014015 hep-ph/0406232];

P. Ball and R. Zwicky, Phys. Rev. D 71 (2005) 014029 [hep-ph/0412079].

[28] F. De Fazio, T. Feldmann and T. Hurth, Nucl. Phys. B 733 (2006) 1 [Erratum-ibid. B 800 (2008) 405] hep-ph/0504088;

F. De Fazio, T. Feldmann and T. Hurth, JHEP 0802 (2008) 031, arXiv:0711.3999 [hep-ph].

[29] M. Beneke, T. Feldmann and D. Seidel, Nucl. Phys. B 612 (2001) 25 hep-ph/0106067;

M. Beneke, T. Feldmann and D. Seidel, Eur. Phys. J. C 41 (2005) 173 hep-ph/0412400].

[30] C. Bobeth, G. Hiller and G. Piranishvili, JHEP 0807 (2008) 106, arXiv:0805.2525 [hep-ph];

U. Egede, T. Hurth, J. Matias, M. Ramon and W. Reece, JHEP 0811 (2008) 032, arXiv:0807.2589 [hep-ph];

U. Egede, T. Hurth, J. Matias, M. Ramon and W. Reece, arXiv:1005.0571 [hep-ph];

W. Altmannshofer, P. Ball, A. Bharucha, A. J. Buras, D. M. Straub and M. Wick, JHEP 0901 (2009) 019, arXiv:0811.1214 [hep-ph];

A. Bharucha and W. Reece, arXiv:1002.4310 [hep-ph].

[31] T. Hurth, Int. J. Mod. Phys. A 22 (2007) 1781 hep-ph/0703226];

M. Artuso et al., Eur. Phys. J. C 57 (2008) 309, arXiv:0801.1833 [hep-ph];

T. Hurth and M. Nakao, arXiv:1005.1224 [hep-ph].

[32] A. F. Falk, M. E. Luke and M. J. Savage, Phys. Rev. D 49 (1994) 3367 hep-ph/9308288;

A. Ali, G. Hiller, L. T. Handoko and T. Morozumi, Phys. Rev. D 55 (1997) 4105 hep-ph/9609449;;

G. Buchalla and G. Isidori, Nucl. Phys. B 525 (1998) 333 hep-ph/9801456].

[33] G. Buchalla, G. Isidori and S. J. Rey, Nucl. Phys. B 511 (1998) 594 hep-ph/9705253. 
[34] K. S. M. Lee, Z. Ligeti, I. W. Stewart and F. J. Tackmann, Phys. Rev. D 75 (2007) 034016 [hep-ph/0612156].

[35] M. Beneke, G. Buchalla, M. Neubert and C. T. Sachrajda, Eur. Phys. J. C 61 (2009) 439, arXiv:0902.4446 [hep-ph].

[36] K. S. M. Lee and I. W. Stewart, Phys. Rev. D 74 (2006) 014005 [hep-ph/0511334; K. S. M. Lee, Z. Ligeti, I. W. Stewart and F. J. Tackmann, Phys. Rev. D 74 (2006) 011501 hep-ph/0512191].

[37] K. S. M. Lee and F. J. Tackmann, Phys. Rev. D 79 (2009) 114021, arXiv:0812.0001 [hep-ph].

[38] M. Beneke, Phys. Lett. B 434 (1998) 115 hep-ph/9804241.

[39] T. Huber, T. Hurth and E. Lunghi, Nucl. Phys. B 802 (2008) 40, arXiv:0712.3009 [hep-ph].

[40] H. M. Asatrian, K. Bieri, C. Greub and A. Hovhannisyan, Phys. Rev. D 66 (2002) 094013 [hep-ph/0209006]. 